\title{
A Novel Spleen Tyrosine Kinase Inhibitor Suppresses Invasiveness of Rheumatoid Arthritis Fibroblast Like Synoviocytes and Improves Collagen Induced Arthritis
}

\section{Seon Uk Kim}

Seoul National University

Hyun Jung Yoo

Seoul National University

Jung Ho Kim

Oscotec Inc.

Hae Jun Hwang

Oscotec Inc.

Jin Kyun Park

Seoul National University Hospital

Eun Young Lee

Seoul National University Hospital

Yeong-Wook Song ( $\nabla$ ysong@snu.ac.kr)

Seoul National University https://orcid.org/0000-0002-5384-3437

Research article

Keywords: Rheumatoid arthritis, inflammation, fibroblast-like synoviocytes, cell migration, signal transduction, spleen tyrosine kinase, spleen tyrosine kinase inhibitor

Posted Date: December 15th, 2020

DOI: https://doi.org/10.21203/rs.3.rs-126063/v1

License: (c) (1) This work is licensed under a Creative Commons Attribution 4.0 International License. Read Full License 


\section{Abstract}

\section{Background/Purpose}

Rheumatoid arthritis (RA) is a chronic inflammatory autoimmune disease characterized by bone and cartilage destruction with leukocyte infiltration and activation at synovial tissue. The fibroblast-like synoviocytes (FLS) have a central role in disease pathogenesis and their invasiveness correlates with articular damage in RA patients. Spleen tyrosine kinase (SYK) is a non-receptor tyrosine kinase known to have a crucial role in immune receptor signaling. This study aimed to evaluate the inhibitory effect of a novel small-molecule SYK inhibitor, SKI-0-592, on the invasiveness of RA FLS and inflammation of monocytes in vitro and in a mouse collagen-induced arthritis (CIA) model in vivo.

Methods

FLS were isolated from synovial tissues of RA patients. FLS were treated with SKI-0-592 for $1 \mathrm{hr}$ and then stimulated with tumor necrosis factor-alpha (TNF-a) for $48 \mathrm{hr}$. After stimulation, cell viability was measured using cell counting kit-8 (CCK-8) assay. The levels of IL-6, IL-8, CXCL10, MMP-3, and TNF-a were measured in culture supernatant of RA FLS and the monocytic cell line THP-1 cells by ELISA. Wound healing assay transwell migration and invasion assay using RA FLS was performed to evaluate cell migration ability. The adhesion ability of FLS was evaluated by co-culture with calcein-AM labeled THP-1 cells, and the expression of VCAM-1, ICAM-1, a-tubulin, $\beta$-actin, total and phosphorylated SYK, c-Jun Nterminal kinase (JNK), p38, ERK, phosphorylated c-Jun, mitogen-activated protein kinase kinase 4 (MKK4), and MKK3/6 was determined by Western blotting. CIA was developed in DBA/1J mice. Clinical arthritis score and histological scores were evaluated after treatment with SKI-0-592.

Results

SKI-0-592 reduced the secretion of chemokine, CXCL10 in RA FLS. Migration of cells to the wound region and through membrane pores and matrigel were decreased by SKI-0-592. Phosphorylation of JNK and p38 was reduced by SKI-0-592 after TNF-a stimulation. SKI-0-592 decreased secretion of TNF-a levels dose-dependently in THP-1 cells with IgG stimulation. The viability and proliferation of FLS and THP-1 were not affected by SKI-0-592. In the CIA model, scores for clinical arthritis and histology were decreased following SKI-0-592 treatment.

Conclusion

SKI-0-592 inhibited the invasiveness of RA FLS and had an anti-inflammatory effect on monocytes. SKI0-592 exhibited therapeutic effects in the mouse CIA model by improving clinical and histological scores with amelioration of joint destruction. In conclusion, a novel SYK inhibitor, SKI-0-592, may provide a new therapeutic option for RA patients.

\section{Background}


Rheumatoid arthritis (RA) is a chronic inflammatory autoimmune disease characterized by bone and cartilage destruction with infiltration of mononuclear cells including T lymphocytes, B lymphocytes, dendritic cells, and macrophages to synovial tissue [1]. The fibroblast-like synoviocytes (FLS) have an important role in disease pathogenesis, and contribute to articular damage in RA patients. FLS in RA synovium plays a crucial role in invasiveness through matrix degradation mediated by metalloproteinase (MMP). They also express pro-inflammatory cytokines such as tumor necrosis factor (TNF), interleukin (IL)-6 and IL-8 [2]. Furthermore, they develop an aggressive phenotype and can migrate and invade cartilage $[3,4]$.

Spleen tyrosine kinase (SYK), an intracellular protein tyrosine kinase, is a key mediator of immunoreceptor signaling in inflammatory cells, including B cells, mast cells, macrophages, and neutrophils [5]. It was reported that SYK expression is upregulated in the intimal lining of synovial tissues of RA patients and FLS exhibit significantly higher levels of activated SYK in RA patients than in OA patients or healthy individuals [6]. Previous studies have reported that excessive SYK activation is associated with autoimmune diseases such as rheumatoid arthritis, asthma, and allergic rhinitis [7]. SYK activation plays an essential role in TNF-a induced cytokine production in FLS [6] and RANKL (receptor activator of nuclear factor kappa-B ligand)-induced osteoclastogenesis [8].

In addition, some studies have shown benefits from SYK inhibition by reporting that SYK inhibitor, R406 (fostamatinib), modulates the maturation and survival of B cells and suppresses pro-inflammatory cytokine production in RA FLS and also prevents joint inflammation and destruction in arthritis models [911]. Other studies have found that SYK deficient(-/-) chimeric mice survive without abnormal development or defects in major organs and prevent bone erosion in arthritis [12]. Treatment with R406, however, showed undesirable side effects including hypertension and neutropenia in a clinical trial for RA, thereby indicating that the selectivity of SYK inhibition needs to be improved [13]. A novel small-molecule SYK inhibitor, SKI-0-592, was designed and synthesized by Oscotec Inc. (Sungnam, Korea) as a drug candidate for RA. It was considered a suitable candidate for pharmaceutical development as a result of its physicochemical properties $[14,15]$.

Originally, SYK was thought to function primarily in signaling of immunoreceptors such as Fc receptor (FcR) and B cell receptor (BCR). However, several studies reported a crucial role for it in the cell signaling of diverse cellular stimuli, including IL-1, TNF-a and lipopolysaccharide (LPS) [16-19]. SYK inhibition also potently inhibited TNF production induced by FcyR cross-linking in macrophages derived from human monocytes and in the monocytic cell line THP-1 [7, 20]

Emerging approaches for RA treatment have focused on targeting key enzymes mediating multiple signal transduction pathways. SYK meets this definition as it is a master regulator of downstream signal transduction cascades that affect diverse biological functions. Transcriptional activation in FLS is mediated by intracellular signal transduction pathways such as mitogen-activated protein kinase (MAPK), activator protein 1 (AP-1), and nuclear factor KB (NF-KB) [21]. Several recent studies have evaluated signal transduction mechanisms in RA FLS responsible for pro-inflammatory mediator release, especially 
MAPKs and their regulation by upstream kinases $[22,23]$. JNK phosphorylation increased the activity of transcription factors such as c-Jun [24]. This pathway plays a critical role in cell proliferation, apoptosis, angiogenesis and migration.

In this study, we aimed to investigate the therapeutic potential of a novel SYK inhibitor, SKI-0-592, by evaluating its effects on cell migration, invasion, and adhesion in RA FLS, the signaling pathway and antiinflammatory effects in monocytes and finally inflammatory arthritis in an in vivo collagen-induced arthritis (CIA) model.

\section{Methods And Materials}

\section{Chemical compounds and reagents}

SYK inhibitor SKI-0-592 was designed and provided by Oscotec Inc. (Sungnam, Korea). This compounds were dissolved in dimethyl sulfoxide (DMSO, Sigma-Aldrich, MO, USA).

\section{Patient samples and cell preparation}

\section{Patient samples}

Synovial tissue of RA patients was obtained during knee replacement surgery at Seoul National University Hospital. All patients fulfilled the classification criteria of the American College of Rheumatology/The European League Against Rheumatism (ACR/EULAR) 2010 [25]. FLS were isolated from synovial tissues of RA patients. After chopping tissues with a sterile surgical blade and forceps, the specimens were incubated at $37^{\circ} \mathrm{C}$ in serum-free DMEM (Welgene, Gyeongsangbuk-do, Korea) with $1 \mathrm{mg} / \mathrm{ml}$ type II collagenase (Worthington Biochemical, $\mathrm{OH}, \mathrm{USA}$ ). Incubated tissues were washed with phosphatebuffered saline (PBS) and passed through a $40 \mu \mathrm{m}$ cell strainer. Filtered cells were cultured in DMEM containing 10\% (V/V) fetal bovine serum (FBS, Gibco, ThermoFisher Scientific, MA, USA) and 1\% penicillin/streptomycin (P/S, Gibco). We used FLS between passages four and eight in experiments. Prepared FLS were pretreated with vehicle (0.1\% DMSO) or SYK inhibitor (SKI-0-592) in serum-free DMEM containing 1\% P/S for 1 hour and stimulated with $10 \mathrm{ng} / \mathrm{ml}$ TNF-a (R\&D systems, MN, USA) for 48 hours. FLS were collected and used for experiments as approved by Seoul National University Hospital Institutional Review Board (SNUH IRB) under protocol no. 1504-086-665.

\section{Cell lines}

THP-1 cells, a human monocyte cell line, were purchased from ATCC. These cells were cultured in RPMI containing 10\% FBS and 1\% P/S with $0.05 \mathrm{mM}$ 2-mercaptoethanol (Gibco), $10 \mathrm{mM}$ HEPES and $1 \mathrm{mM}$ sodium pyruvate until used.

\section{Cell viability assay}


For assessment of cytotoxicity, we examined cell viability after treatment with drug using cell counting kit-8 solution (CCK-8, Dojindo, Kumamoto, Japan). CCK-8 was added to each well of cells treated, and then incubated for 1 hour 30 minutes at $37^{\circ} \mathrm{C}$ in the dark, after which we measured the absorbance at $450 \mathrm{~nm}$ using a microplate reader (Versamax, Molecular Devices, CA, USA).

\section{Cytokines, chemokines and MMPs assay}

The concentration of IL-6, IL-8, MMP-3, and C-X-C motif chemokine 10 (CXCL10) were measured from culture supernatants of FLS. The level of TNF-a was measured from culture supernatants of THP-1 cells. All analyses except TNF-a were performed using DuoSet enzyme-linked immunosorbent assay (ELISA) kit (R\&D Systems). TNF-a levels were measured by human TNF-a cytokine ELISA kits (BD Biosciences). Antibody array was performed using a human inflammation antibody array kit (Abcam, Cambridge, UK). The array was analyzed using the same methods as a chemiluminescent immunoblot. A comparison between samples was done by using densitometry software. All analyses were performed according to the manufacturer's instructions.

\section{Immunoblot analysis}

FLS were lysed into cell lysis buffer containing $20 \mathrm{mM}$ Hepes-NaOH (pH 7.0), $150 \mathrm{mM} \mathrm{NaCl,} 1 \mathrm{mM}$ EDTA, $1 \mathrm{mM}$ EGTA, $1 \mathrm{mM}$ sodium vanadate, $10 \mathrm{mM}$-phosphoglycerate, 1\% Nonidet P-40, 10\% glycerol, $1 \mathrm{mM}$ trichostatin A, $5 \mathrm{mM} \mathrm{NaF}$ and $20 \mathrm{mM}$ nicotinamide, along with Halt protease and phosphatase inhibitor cocktail (ThermoFisher Scientific) after washing once with PBS. The amount of protein was quantified with Bradford protein assay (Bio-Rad, CA, USA). The same amounts of total protein lysate were subjected to Bolt 4-12\% bis-tris plus gels (Invitrogen, ThermoFisher Scientific) and separated by electrophoresis. Following protein electrotransfer the nitrocellulose membranes were blocked with $5 \%$ skim milk for 30 min in tris-buffered saline containing 0.05\% Tween 20 (TBST). Blocked membranes were washed with TBST and incubated overnight at $4{ }^{\circ} \mathrm{C}$ with primary antibodies. We used antibodies recognizing phosphorylated mitogen-activated protein kinase kinase 4 ( $p-M K K 4)$, MKK3/6, JNK, p-JNK, p38, p-p38, ERK, p-ERK, p-C-Jun, $\beta$-actin and a-tubulin (Cell Signaling Technology, MA, USA), ICAM-1 and VCAM-1 (Santa Cruz, TX, USA). After incubation, the membranes were washed with TBST and incubated with the horseradish peroxidase-conjugated anti-mouse or anti-rabbit immunoglobulin (secondary antibodies, Jackson Immuno Research Laboratories, PA, USA) for 1 hour at room temperature. Membrane-bound secondary antibodies were detected by a chemiluminescent substrate (Elpis Biotech, Daejeon, Korea). Films were scanned, and band densities were quantified using ImageJ software.

\section{Wound healing assay}

After seeding $1 \times 10^{4}$ cells/ml of FLS in 6-well culture plates, cell monolayers were scraped using a sterile pipette tip and detached cells were washed out. The wounded cell layers were left untreated or pretreated with $10 \mu \mathrm{M}$ SKI-0-592. Images of wounded sites were taken 16 hours and 24 hours after treatment using phase-contrast microscopy on a DFC295 (Leica, Wetzlar, Germany) with LAS V3.8 software (Leica). Cells beyond specified guidelines in three randomized sites were counted for quantification of data. 


\section{Migration assay}

To determine the impact of SKI-0-592 on migration of FLS, $2 \times 10^{4}$ cells were seeded in Corning Transwell polycarbonate $8 \mu \mathrm{m}$ pore membrane cell culture inserts for 24-well plates (Corning, New York, USA) containing a final concentration of $0.5 \%$ FBS. The lower chamber contained $20 \mathrm{ng} / \mathrm{ml}$ TNF-a as a chemoattractant. After incubation for $24 \mathrm{hrs}$, the non-migrated cells on top of the membrane were removed by gentle wiping with a cotton-tipped swab. The migrated cells on the lower surface of the membrane were stained with $0.1 \%$ crystal violet dye, and FLS were counted in six representative areas of three wells (200x magnification).

\section{Invasion assay}

To determine the impact of SKI-0-592 on FLS invasiveness, each well was coated with $300 \mu \mathrm{g} / \mathrm{ml}$ of matrigel (Corning) and $4 \times 10^{4}$ cells were seeded as described above and but with $10 \% \mathrm{FBS}$. The lower chamber contained $20 \mathrm{ng} / \mathrm{ml} \mathrm{TNF}-\mathrm{a}$ as a chemoattractant. After incubation for $72 \mathrm{hrs}$, the non-invaded cells on top of the membrane were removed by gentle wiping with a cotton-tipped swab. The invaded cells on the lower surface of the membrane were stained with $0.1 \%$ crystal violet dye, and FLS were counted in six representative areas of three wells (200x magnification).

\section{Adhesion assay}

THP- 1 cells were labeled with $5 \mu \mathrm{M}$ calcein-AM (Thermofisher Scientific) at $37^{\circ} \mathrm{C}$ for 30 minutes. Calceinlabeled cells were washed with pre-warmed serum-free RPMI and resuspended at a density of $2 \times 10^{6}$ cells $/ \mathrm{ml}$. Resuspended cells were added to untreated or drug-treated RA FLS with $1 \mathrm{hr}$ pre-treatment of SKI-O-592 and incubated at $37^{\circ} \mathrm{C}$ for $30 \mathrm{~min}$. Unattached cells in each well were removed by washing once with PBS. Fluorescent images of THP-1 cells adhering to RA FLS were observed and analyzed at an excitation wavelength of $494 \mathrm{~nm}$ and an emission wavelength of $517 \mathrm{~nm}$. The number of THP-1 cells adhering to FLS were counted in five representative areas of each well (100x magnification).

\section{TNF- $a$ release in THP-1 cell lines}

THP-1 cells were maintained as described above. For the TNF-a release assay, $1 \times 10^{5}$ THP-1 cells were plated in human IgG $(100 \mu \mathrm{g} /$ well $)$ pre-coated 96 -well plates and then treated with the indicated concentrations of SYK inhibitors. After incubation for 24 hours, the TNF-a level in supernatants was measured by ELISA and the remaining cells were subjected to a CCK-8 assay to determine the cytotoxic effects of SYK inhibitors. For ELISA, anti-hTNF-a (R\&D systems) was pre-coated, and then streptavidin HRP (R\&D systems) and TMB substrate (BD Biosciences) were used. To investigate the effect on SYKindependent TNF-a release, THP-1 cells were treated with LPS $(1 \mu \mathrm{g} / \mathrm{mL})$ and the indicated concentrations of SYK inhibitors simultaneously.

\section{Measurement of FcyR-mediated response by Western blot}


THP-1 cells were incubated with SYK inhibitors for $1 \mathrm{hr}$ and then stimulated with human $\operatorname{lgG}(100 \mu \mathrm{g} / \mathrm{mL})$ for 15 minutes. Cells were lysed in RIPA buffer [ $25 \mathrm{mM}$ Tris-HCl pH 7.6, $150 \mathrm{mM} \mathrm{NaCl}, 1 \%$ nonyl phenoxypolyethoxylethanol, $1 \%$ sodium deoxycholate, $0.1 \%$ SDS] containing protease and phosphatase inhibitor cocktail (Thermo Scientific). To examine phosphorylation levels, whole cell lysates were subjected to immunoblotting. Equivalent amounts of protein were separated by NuPAGE $4-12 \%$ bis-tris gel system (Invitrogen) and then transferred to polyvinylidene difluoride membranes. Membranes were probed with an anti-phospho-SYK (Y525/526), anti-phospho-SYK (Y352), or anti-phospho-VAV (Y174). After incubation with the secondary antibody, the membranes were visualized by enhanced chemiluminescence with the Pierce ECL plus substrate.

\section{Induction of collagen-induced arthritis in mice}

DBA1/J male (6-7 weeks old) mice were injected intradermally with $0.1 \mathrm{ml}$ bovine type $\otimes$ collagen (100 $\mu \mathrm{g}$; Chondrex Inc., WA, USA) and complete Freund's adjuvant ( $200 \mu \mathrm{g}$; Difco, MI, USA) followed by second immunization on day 21 with bovine type $\otimes$ collagen and incomplete Freund's adjuvant [26]. The vehicle was composed of N-methyl-2-pyrrolidone (NMP), polyethylene glycol 400 (PEG400), 2-hydroxypropylbeta-cyclodextrin (HPBCD) in proportions of 5:50:45. Mice were randomized into four different groups (10 mice/group) and SKI-0-592 was diluted with a vehicle and administered orally every day for 14 days starting on the day after second immunization. Joint sections from animals treated with vehicle and drugs were stained with hematoxylin and eosin (H\&E) or Safranin-O solution. Clinical arthritis scores ( 0 to 4) of individual paws were assessed, and the arthritic index for each mouse was determined by adding the individual scores of all four paws. Clinical scores were monitored on days 1, 5, 7, 11, 14, 18, and 20. For arthritis score, the edema and swelling of each paw were scored visually, using a scale of $0-4$, where $0=$ no visible abnormalities, $1=$ one or two swelling digits, $2=$ three or more than three swelling digits or swelling from the ankle to the entire paw, $3=$ one or two swelling digits and swelling from the ankle to the entire paw, 4=three or more than three swelling digits and swelling from the ankle to the entire paw.

\section{Statistical analysis}

All data are presented as mean \pm SEM or SD. We used the 2-tailed Student's t-test for in vitro data to evaluate differences between two groups, and $\mathrm{P}<0.05$ was considered statistically significant. Two-way ANOVA with Bonferroni correction was used for statistical analysis of in vivo data. Statistical analyses were performed using GraphPad Prism 5.01 (GraphPad, CA, USA).

\section{Results}

\section{SKI-0-592 inhibits SYK phosphorylation in RA FLS.}

We assessed the expression of SYK and phosphorylated SYK using Western blot on RA FLS after SKI-O592 treatment with subsequent TNF-a stimulation (Figure 1A). SKI-0-592 decreased levels of phosphorylated SYK. To exclude the potential cytotoxic effects of SKI-0-592 on FLS, cell viability assays were performed using CCK-8 assay. The viability of RA FLS were not affected by doses of SKI-0-592 up to 
$10 \mu \mathrm{M}$ (Figure 1B). These data showed that SKI-0-592 is an SYK inhibitor that inhibits phosphorylation without cell toxicity.

\section{Effect of SKI-0-592 on the production of cytokines, chemokine, and MMP in RA FLS}

We examined whether SKI-0-592 affects the secretion of pro-inflammatory molecules in RA FLS. RA FLS was pretreated with SYK inhibitor and then stimulated with TNF-a for 48 hours. To evaluate the change of various pro-inflammatory molecules, antibody arrays were performed on culture supernatants (Figure 2A). The relative production of pro-inflammatory molecules was analyzed by optical intensity and normalized to a positive control (Figure 2B). The relative secretion of IL-8, CXCL10, monocyte chemoattractant protein-1 (MCP-1), RANTES [chemokine (C-C motif) ligand 5, CCL5], and tissue inhibitor of metalloproteinases-2 (TIMP-2) were all reduced in the SKI-0-592-treated group. Next we measured the concentration of IL-6, IL-8, CXCL10, and MMP-3 in the culture supernatants of RA FLS by ELISA. In SKI-0592-treated RA FLS, the concentration of CXCL10 in the culture supernatants of RA FLS was significantly decreased (Figure 2E). However, SKI-0-592 did not affect the secretion of IL-6, IL-8, or MMP-3 (Figure 2C, $D$ and $F$ ).

\section{SKI-0-592 suppresses RA FLS invasiveness.}

We investigated whether cell migration is regulated by SKI-0-592 in RA FLS using wound healing, transwell migration and invasion assay. In the wound healing assay, cells treated with SKI-0-592 migrated much less than control cells treated with vehicle (Representative image, Figure $3 \mathrm{~A}$ ), and the number of cells that migrated to the wound region at 16 and $24 \mathrm{hr}$ was significantly lower in SKI-0-592treated cells than in control cells (Figure 3B). In the transwell migration assay with crystal violet staining, fewer cells migrated through the membrane to the lower surface of inserts (Representative image, Figure 3C), and the number of migrated cells in SKI-0-592-treated wells was significantly lower than the control wells (Figure 3D). Moreover, the transwell invasion assay showed the effect of SKI-0-592 on the cell invasion capability of RA FLS. Fewer cells invaded across the matrigel membrane to the lower surface of the inserts in SKI-0-592-treated cells (Representative image, Figure 3E), and the number of invaded cells seen with crystal violet staining in SKI-0-592-treated wells was significantly lower than the control wells (Figure 3F). These results indicate that SKI-0-592 markedly inhibits the invasiveness of FLS from RA patients.

\section{SKI-0-592 represses cell adhesion and downregulates the expression of adhesion molecules.}

THP-1 cells were used for the assessment of cell adhesion to RA FLS. TNF-a significantly increased THP1 cell adhesion, which was inhibited by SKI-0-592 treatment (Figure 4A, B). In RA FLS, TNF-a-induced expression of VCAM-1 was dose-dependently reduced by SKI-0-592 but ICAM-1 was not affected (Figure 4C-E). SKI-0-592 may control leukocyte adhesion to RA FLS through inhibiting expression of adhesion molecules VCAM-1 and chemokine CXCL10, suggesting that SKI-O-592 reduces leukocyte infiltration to RA synovial tissue. 


\section{Inhibitory effect of SKI-0-592 on the phosphorylation of MAPKs and c-Jun.}

Given that TNF-a is a potent activator of MAPK, we performed Western blot analysis to determine the effect of SYK inhibition on MAPK phosphorylation. SKI-0-592 decreased TNF-a-induced phosphorylation of JNK and p38 (Figure 5A, B). However, ERK phosphorylation was not affected by SKI-0-592 treatment. Then, we investigated the effect of SKI-O-592 on the activation of the upstream MAPK kinases MKK4 and MKK3/6, which activate JNK and p38. MKK4 and MKK3/6 phosphorylation peaked 15 min after TNF-a stimulation, and this was inhibited by SKI-O-592 (Figure 5C, D). To evaluate the functional relevance of SYK-mediated JNK and p38 activation, the effect of SYK inhibition on the JNK substrate c-Jun was evaluated. C-Jun phosphorylation peaked $15 \mathrm{~min}$ after TNF-a stimulation and was significantly suppressed by SKI-0-592 (Figure 5C, D). These data indicate that SYK plays a crucial role in both JNK and p38 activation in synoviocytes.

\section{Phosphorylation of MAPKs was inhibited dose-dependently by SKI-0-592.}

To examine dose-dependent inhibitory effects of SKI-0-592 in kinase phosphorylation, we treated with various concentrations of the drug simultaneously with $10 \mathrm{ng} / \mathrm{ml}$ TNF-a stimulation for $15 \mathrm{~min}$ (Figure 6A). SKI-0-592 suppressed p54 JNK phosphorylation dose-dependently (Figure 6B). p46 JNK was not affected. Dose-dependent inhibition was also observed on p38 phosphorylation by SKI-0-592 treatment (Figure $6 \mathrm{C}$ ). These results demonstrated that phosphorylation of JNK and p38 was dose-dependently suppressed by SKI-0-592.

\section{Inhibitory effect of SKI-0-592 on IgG induced TNF-a production and FcyR-mediated pathways in monocytes.}

SKI-0-592 inhibited the phosphorylation of SYK at Tyr525/526 (Figure 7A, B) and slightly inhibited phosphorylation at Tyr352 (Figure 7C). It suppressed downstream effector, VAV phosphorylation, suggesting that SKI-O-592 inhibited SYK in monocytes (Figure 7D). TNF-a was measured in the culture supernatant of THP-1 cell after IgG stimulation compared to LPS stimulation (Figure 7E). Under IgG stimulation, SKI-0-592 inhibited the release of TNF-a with $\mathrm{IC}_{50}$ values of $45 \mathrm{nM}$ in THP-1 cells. Upon LPS stimulation, SKI-0-592 up to a concentration of $10 \mu \mathrm{M}$ did not inhibit the level of TNF-a. It also did not affect cell viability even up to $10 \mu \mathrm{M}$, suggesting that the anti-inflammatory activity is not caused by cytotoxic activity against cells (Figure 7F). These data indicate that SKI-0-592 is efficacious for the inhibition of TNF-a release through SYK-dependent inflammatory signals in immune receptor-mediated responses.

\section{SKI-0-592 reduced joint destruction and arthritis clinical scoring in mouse CIA.}

Based on the findings thus far that SYK inhibition inhibited several innate and adaptive immune responses, we assumed that the compound might have therapeutic efficacy in an in vivo inflammatory arthritis model. Therefore we induced CIA in DBA/1J mice. Vehicle or SKI-0-592 was administered orally every day after the second immunization at a dose of $30 \mathrm{mg} / \mathrm{kg} /$ day (ten animals per group). The 
arthritis clinical score was significantly diminished in the SKI-0-592 administered group compared to the vehicle treated group (Figure $8 \mathrm{~A}$ ). In the histopathological analysis, mice had reduced inflammatory cell infiltration in the joint tissues and cartilage damage in the ankle and had significantly lower histological scores (Figure 8B-D).

\section{Discussion}

Rheumatoid arthritis (RA) is a chronic inflammatory autoimmune disease characterized by bone and cartilage destruction with leukocyte infiltration and activation at synovial tissue. Pathogenesis involves the production of pro-inflammatory mediators such as cytokines, chemokines and MMPs and depending upon which, these recruit cells to the joint and the extracellular matrix [1]. SYK is a key mediator of immunoreceptor signaling in inflammatory cells, including B cells, mast cells, macrophages, and neutrophils [5], and is thought to function primarily in signaling of immunoreceptors such as Fc receptor $(\mathrm{FcR})$ and $\mathrm{B}$ cell receptor (BCR).

In addition, some studies have shown that SYK inhibition modulates the maturation and survival of B cells and suppresses pro-inflammatory cytokine production in RA FLS and also prevents joint inflammation and destruction in arthritis models [9-11]. SYK(-/-) macrophages and neutrophils exhibit defective generation of reactive oxygen intermediates and phagocytosis in response to integrin or FcyR engagement [27]. SYK-deficient dendritic cells failed to internalize antigens or efficiently stimulate $T$ cells after exposure to immune complexes [28]. SYK mutations impair the differentiation of B lymphocytes by disrupting signaling from the pre-BCR complex thereby preventing the clonal expansion of pre-B cells [29]. In the same study, SYK also mediated immunoreceptor-like signals required for the development and function of osteoclasts, and hematopoietic SYK deficiency completely blocks the development of osteoclasts $[12,30]$.

Testing of a SYK inhibitor however, showed unexpected side effects including hypertension and neutropenia in a clinical trial for RA, indicating that the selectivity of SYK inhibitors needs to be improved [13]. SKI-0-592 was designed and synthesized as a novel SYK inhibitor. In the previous study, SKI-0-592 had a lower $\mathrm{IC}_{50}$ value than fostamatinib on the kinase activity of SYK with lower inhibition of JAK2, JAK3, and KDR (vascular endothelial growth factor receptor 2, VEGFR-2), which is the primary cause of side effects such as hypertension [31].

FLSs play an essential role in disease pathogenesis, They play the central role in invasiveness with matrix degradation occurring through production of metalloproteinase, and they also express pro-inflammatory cytokines such as IL-6 and TNF-a [2, 32]. FLS in RA are not simple passive responders that react to the inflammatory milieu but are also epigenetically imprinted with an activated and aggressive phenotype that operates independently of the inflammatory stimuli [33]. Fostamatinib, a first-in-class SYK inhibitor, attenuated synovial inflammation in rodent arthritis models and reduced the production of inflammatory cytokines and proteases such as IL-6 and MMP-3 in synovium-derived RA FLS [6, 7]. 
RA FLS become hyperplastic and increase their migration and mobility, invading the cartilage and destroying it. Cell adhesion is associated with cell migration [34]. The specific sequences of cellular events and interactions leading to progressive destruction of articular structures have not been fully clarified. p38/JNK signaling has been shown to participate in the modulation of migration processes in various cells [34]. However, the association between the p38/JNK signaling pathway and migration of RA FLS was unclear. Our results revealed that SKI-0-592 significantly reduced the invasive capacity of RA FLS. SKI-0-592 reduced the phosphorylation of p38 and JNK in RA FLS and these results suggest that SYK inhibition specifically affects the p38/JNK signaling pathway. Clinically, the invasiveness of synovial lining cells was sustained despite the amelioration of inflammation. Therefore, inhibition of RA FLS migration may serve as an important target for RA therapies [33].

The three MAPK families ERK, JNK, and p38, phosphorylate numerous key transcription factors, such as AP-1 and ATF-1/2 [35]. MAPKs are especially important because they control the production of MMPs and cytokines that participate in the rheumatoid process. The JNKs are activated by dual phosphorylation of upstream kinases (MKK4 and MKK7) and are highly activated in isolated RA FLS or the rheumatoid synovial lining layer with synovial mononuclear cell infiltrates. Downstream components to the AP-1 complex, c-Jun, and c-Fos are also expressed in both the synovial lining and sub-lining layer [24]. Activation of the JNK pathway following exposure to pro-inflammatory cytokines, such as TNF-a and IL-1, plays a crucial role in many kinds of cellular behaviors, such as cell migration, proliferation, differentiation, apoptosis and stress reaction. The p38 MAPK signal transduction pathway is reported to be a key regulator of IL-1 and TNF-a production in rheumatoid arthritis. Previous studies also demonstrated that upstream MAPK kinases (MKK3 and MKK6) that regulate p38 are activated in the rheumatoid arthritis synovium [36].

The present data suggest that SYK regulates the MAPK cascade in RA FLS, particularly through the JNK, and p38 pathways. Blocking of SYK resulted in inhibition of TNF-a-induced JNK and p38 phosphorylation. Phosphorylation of the upstream MAPK kinases MKK4 and MKK3/6 was decreased. MKK4 can also phosphorylate p38 MAPKs under some circumstances [24]. It was reported that MKK4 is primarily activated by environmental stress and preferentially phosphorylates tyrosine $(\mathrm{Y})$ residue 185 in the p54 site of JNK1, whereas MKK7, preferentially phosphorylates threonine (T) residue 183 in the p46 site. This is consistent with the observation that p54 was inhibited by SKI-0-592. This result suggests that MAPKs inhibition was not likely the result of a direct action of the compound, but that SYK affects MAPK kinase proximal to JNK or p38 pathways.

The functional relevance of JNK inhibition in RA FLS was confirmed by demonstrating downstream effects on c-Jun phosphorylation. JNK activation was reported to be associated with transcription factor AP-1 activation, and expression of pro-inflammatory cytokines. The importance of SYK in TNF-induced JNK activation was also highlighted in an earlier study in which they showed that pretreatment with piceatannol, a naturally occurring SYK inhibitor, induced a concentration-dependent inhibition of JNK1 activation in stimulated neutrophils [37]. 
In contrast to JNK and p38, SYK inhibitor had little or no effect on the ERK pathway in cultured FLS. These results contrast with studies in Jurkat cells, where piceatannol completely suppressed TNFinduced activation of all three MAPKs [19]. Although the effect of SKI-0-592 is likely mediated through selective inhibition of SYK, it is possible that the compound also affects other kinases. In the previous study, the compound fostamatinib potentially inhibits kinases such as JAK2, JAK3, and KDR, which are related to side effects such as hypertension [38], but SKI-0-592 had only weak inhibitory effects on those kinases (unpublished data).

Members that are upstream in the kinase cascade of the MAPK family, such as MKK3, MKK6 and MAP3K5 (also ASK1, apoptosis signal-regulating kinase 1), are alternative targets that have been explored in preclinical models. ASK1 is hypomethylated in RA FLS compared to OA FLS and proinflammatory cytokines induce the expression of ASK1. Treatment with a small-molecule inhibitor of ASK1 reduced invasion, migration and proliferation of RA FLS in vitro. Furthermore, MAP3K5 ${ }^{-/-}$mice were protected from K/BxN serum-induced arthritis, and treatment with MAP3K5 inhibitor also protected against joint damage and inflammation in rats with collagen-induced arthritis [33].

We did not clarify the mechanisms associated with SYK inhibitor-dependent regulation of adhesion molecules. In a previous study, upregulated production of VCAM-1 and ICAM-1 by TNF-a were restored in the presence of MAPK inhibitors and NF-KB inhibitor, suggesting a potential connection between adhesion molecules and MAPK or NF-KB signaling, especially via p38 MAPK signaling pathway $[39,40]$. The migration of leukocytes into infected or injured sites is an essential step for primary immune responses. Leukocytes recruited to inflamed tissues, play a central role in the progress of RA [34]. Increased expression of adhesion molecules on RA FLS could lead to higher infiltration of immune cells into the inflammatory site. Taken together, we can speculate that SKI-0-592 regulates adhesion ability through inhibition of p38 phosphorylation.

In summary, the novel and selective SYK inhibitor, SKI-0-592, had an impact on invasiveness of RA FLS through the inhibition of cell migration, invasion, and adhesiveness as well as through significant downregulation of chemokines. The mechanism behind these actions may involve the MAPK cascade, in particular inhibition of phosphorylation of JNK and p38. SKI-0-592 also had an anti-inflammatory effect on monocytes by inhibiting the production of TNF-a in THP-1 cells. SKI-0-592 inhibited the phosphorylation of SYK at Tyr525/526 and Tyr352 and also of downstream effector, VAV. These data suggest that SYK serves as a regulator of immune responses in RA FLS and monocytes.

\section{Conclusions}

SKI-0-592 showed a therapeutic effect on the mouse CIA model by improving clinical scores and ameliorating joint destruction. The mechanism behind these effects may be through inhibition of the MAPK cascade, especially JNK and p38. In conclusion, a novel SYK inhibitor, SKI-0-592, may provide a new therapeutic option for RA patients by inhibiting invasiveness of RA FLS and pro-inflammatory functions in monocytes. 


\section{Abbreviations}

ACR: American College of Rheumatology; CCK-8: Cell-counting kit 8; CIA: Collagen-induced arthritis; DMSO: Dimethyl sulfoxide; ELISA: Enzyme-linked immunosorbent assay; FBS: Fetal bovine serum; FLS: Fibroblast-like synoviocytes; IFN: Interferon; IL: Interleukin; JNK: c-Jun N-terminal kinase; LPS:

Lipopolysaccharide; MAPK: Mitogen-activated protein kinase; MKK: Mitogen-activated protein kinase kinase; MMP: Metalloproteinase; P/S: Penicillin/streptomycin; PBS: Phosphate buffered saline; RA: Rheumatoid arthritis; RPMI: RPMI 1640; Secondary antibody, Horseradish peroxidase-conjugated antimouse or anti-rabbit immunoglobulin; TBST: Tris-buffered saline containing $0.05 \%$ Tween; TNF: Tumor necrosis factor

\section{Declarations}

\section{Acknowledgements}

Not applicable.

\section{Authors' contributions}

SU Kim performed most of the experiments and wrote the manuscript; HJ Yoo participated in writing and in-vitro experiments; JH Kim and HJ Hwang developed SKI-0-592; JK Park and EY Lee participated in the design of the study; and YW Song contributed to design and manuscript preparation as the corresponding author.

\section{Funding}

This study was supported by a grant from the Ministry of Science, ICT and Future Planning, Republic of Korea (grant number: NRF-2019M3A9A8065574 and NRF-2020M3E2037430). This study was also supported by a grant from Korea Health Technology R\&D Project through the Korea Health Industry Development Institute (KHIDI), funded by the Ministry of Health \& Welfare, Republic of Korea (grant number: HI14C1277).

\section{Availability of data and materials}

Not applicable.

\section{Ethics approval and consent to participate}

Informed consent was obtained from all patients, and the experimental protocol was approved by Seoul National University Hospital Institutional Review Board (SNUH IRB) under protocol no. 1504-086-665.

\section{Consent for publication}

Not applicable. 


\section{Competing interests}

Authors declare no competing interest.

\section{Author details}

${ }^{1}$ Department of Molecular Medicine and Biopharmaceutical Sciences, Graduate School of Convergence Science and Technology, Seoul National University, Seoul, 03082, Korea. ${ }^{2}$ Oscotec Inc., Seongnam, 13488, Korea. ${ }^{3}$ Division of Rheumatology, Department of Internal Medicine, Seoul National University Hospital, Seoul, 03080, Korea

\section{References}

1. Smolen JS, Steiner G: Therapeutic strategies for rheumatoid arthritis. Nat Rev Drug Discov 2003, 2(6):473-488.

2. Firestein GS: Evolving concepts of rheumatoid arthritis. Nature 2003, 423(6937):356-361.

3. Korb-Pap A, Bertrand J, Sherwood J, Pap T: Stable activation of fibroblasts in rheumatic arthritiscauses and consequences. Rheumatology (Oxford) 2016, 55(suppl 2):ii64-ii67.

4. Lefevre S, Knedla A, Tennie C, Kampmann A, Wunrau C, Dinser R, Korb A, Schnaker EM, Tarner IH, Robbins PD et al: Synovial fibroblasts spread rheumatoid arthritis to unaffected joints. Nat Med 2009, 15(12):1414-1420.

5. Wong BR, Grossbard EB, Payan DG, Masuda ES: Targeting Syk as a treatment for allergic and autoimmune disorders. Expert Opin Investig Drugs 2004, 13(7):743-762.

6. Cha HS, Boyle DL, Inoue T, Schoot R, Tak PP, Pine P, Firestein GS: A novel spleen tyrosine kinase inhibitor blocks c-Jun N-terminal kinase-mediated gene expression in synoviocytes. J Pharmacol Exp Ther 2006, 317(2):571-578.

7. Braselmann S, Taylor V, Zhao H, Wang S, Sylvain C, Baluom M, Qu K, Herlaar E, Lau A, Young C et al: $\mathrm{R} 406$, an orally available spleen tyrosine kinase inhibitor blocks fc receptor signaling and reduces immune complex-mediated inflammation. J Pharmacol Exp Ther 2006, 319(3):998-1008.

8. Mocsai A, Ruland J, Tybulewicz VL: The SYK tyrosine kinase: a crucial player in diverse biological functions. Nat Rev Immunol 2010, 10(6):387-402.

9. Pine PR, Chang B, Schoettler N, Banquerigo ML, Wang S, Lau A, Zhao F, Grossbard EB, Payan DG, Brahn E: Inflammation and bone erosion are suppressed in models of rheumatoid arthritis following treatment with a novel Syk inhibitor. Clin Immunol 2007, 124(3):244-257.

10. Liao C, Hsu J, Kim Y, Hu DQ, Xu D, Zhang J, Pashine A, Menke J, Whittard T, Romero N et al: Selective inhibition of spleen tyrosine kinase (SYK) with a novel orally bioavailable small molecule inhibitor, R09021, impinges on various innate and adaptive immune responses: implications for SYK inhibitors in autoimmune disease therapy. Arthritis Res Ther 2013, 15(5):R146. 
11. Tan SL, Liao C, Lucas MC, Stevenson C, DeMartino JA: Targeting the SYK-BTK axis for the treatment of immunological and hematological disorders: recent progress and therapeutic perspectives. Pharmacol Ther2013, 138(2):294-309.

12. Jakus Z, Simon E, Balazs B, Mocsai A: Genetic deficiency of Syk protects mice from autoantibodyinduced arthritis. Arthritis Rheum 2010, 62(7):1899-1910.

13. Ferguson GD, Delgado M, Plantevin-Krenitsky V, Jensen-Pergakes K, Bates RJ, Torres S, Celeridad M, Brown H, Burnett K, Nadolny L et al: A Novel triazolopyridine-based spleen tyrosine kinase inhibitor that arrests joint inflammation. PLoS One 2016, 11(1):e0145705.

14. Coffey G, DeGuzman F, Inagaki M, Pak Y, Delaney SM, Ives D, Betz A, Jia ZJ, Pandey A, Baker D et al: Specific inhibition of spleen tyrosine kinase suppresses leukocyte immune function and inflammation in animal models of rheumatoid arthritis. J Pharmacol Exp Ther 2012, 340(2):350-359.

15. Dowty ME, Jesson MI, Ghosh S, Lee J, Meyer DM, Krishnaswami S, Kishore N: Preclinical to clinical translation of tofacitinib, a Janus kinase inhibitor, in rheumatoid arthritis. J Pharmacol Exp Ther 2014, 348(1):165-173.

16. Ulanova M, Puttagunta L, Marcet-Palacios M, Duszyk M, Steinhoff U, Duta F, Kim MK, Indik ZK, Schreiber AD, Befus AD: Syk tyrosine kinase participates in beta1-integrin signaling and inflammatory responses in airway epithelial cells. Am J Physiol Lung Cell Mol Physiol 2005, 288(3):L497-507.

17. Yamada T, Fujieda S, Yanagi S, Yamamura H, Inatome R, Yamamoto H, Igawa H, Saito H: IL-1 induced chemokine production through the association of Syk with TNF receptor-associated factor- 6 in nasal fibroblast lines. J Immunol 2001, 167(1):283-288.

18. Arndt PG, Suzuki N, Avdi NJ, Malcolm KC, Worthen GS: Lipopolysaccharide-induced C-Jun NH2terminal kinase activation in human neutrophils: role of phosphatidylinositol 3-Kinase and Sykmediated pathways. J Biol Chem 2004, 279(12):10883-10891.

19. Takada Y, Aggarwal BB: TNF activates Syk protein tyrosine kinase leading to TNF-induced MAPK activation, NF-kappaB activation, and apoptosis. J Immunol 2004, 173(2):1066-1077.

20. Turner M, Schweighoffer E, Colucci F, Di Santo JP, Tybulewicz VL: Tyrosine kinase SYK: essential functions for immunoreceptor signalling. Immunol Today 2000, 21(3):148-154.

21. Sweeney SE, Firestein GS: Signal transduction in rheumatoid arthritis. Curr Opin Rheumato/ 2004, $16(3): 231-237$.

22. Sundarrajan M, Boyle DL, Chabaud-Riou M, Hammaker D, Firestein GS: Expression of the MAPK kinases MKK-4 and MKK-7 in rheumatoid arthritis and their role as key regulators of JNK. Arthritis Rheum 2003, 48(9):2450-2460.

23. Hammaker DR, Boyle DL, Chabaud-Riou M, Firestein GS: Regulation of c-Jun N-terminal kinase by MEKK-2 and mitogen-activated protein kinase kinase kinases in rheumatoid arthritis. J Immunol 2004, 172(3):1612-1618.

24. Guma M, Firestein GS: c-Jun N-Terminal Kinase in Inflammation and Rheumatic Diseases. Open Rheumatol J 2012, 6:220-231. 
25. Kay J, Upchurch KS: the American College of Rheumatology/The European League Against Rheumatism (ACR/EULAR) 2010 rheumatoid arthritis classification criteria. Rheumatology 2012, 51(suppl 6):vi5-vi9.

26. Schubert D, Maier B, Morawietz L, Krenn V, Kamradt T: Immunization with glucose-6-phosphate isomerase induces $\mathrm{T}$ cell-dependent peripheral polyarthritis in genetically unaltered mice. $\mathrm{J}$ Immunol 2004, 172(7):4503-4509.

27. Mocsai A, Zhou M, Meng F, Tybulewicz VL, Lowell CA: Syk is required for integrin signaling in neutrophils. Immunity 2002, 16(4):547-558.

28. Sedlik C, Orbach D, Veron P, Schweighoffer E, Colucci F, Gamberale R, loan-Facsinay A, Verbeek S, Ricciardi-Castagnoli $\mathrm{P}$, Bonnerot $\mathrm{C}$ et al: A critical role for Syk protein tyrosine kinase in Fc receptormediated antigen presentation and induction of dendritic cell maturation. J Immunol 2003, 170(2):846-852.

29. Cheng AM, Rowley B, Pao W, Hayday A, Bolen JB, Pawson T: Syk tyrosine kinase required for mouse viability and B-cell development. Nature 1995, 378(6554):303-306.

30. Csete D, Simon E, Alatshan A, Aradi P, Dobo-Nagy C, Jakus Z, Benko S, Gyori DS, Mocsai A: Hematopoietic or Osteoclast-Specific Deletion of Syk Leads to Increased Bone Mass in Experimental Mice. Front Immunol 2019, 10:937.

31. Skinner M, Philp K, Lengel D, Coverley L, Lamm Bergstrom E, Glaves P, Musgrove H, Prior H, Braddock $\mathrm{M}$, Huby R et al: The contribution of VEGF signalling to fostamatinib-induced blood pressure elevation. Br J Pharmacol 2014, 171(9):2308-2320.

32. Bartok B, Firestein GS: Fibroblast-like synoviocytes: key effector cells in rheumatoid arthritis. Immunol Rev 2010, 233(1):233-255.

33. Nygaard G, Firestein GS: Restoring synovial homeostasis in rheumatoid arthritis by targeting fibroblast-like synoviocytes. Nat Rev Rheumatol 2020, 16(6):316-333.

34. Luan L, Ma Y, Zhang L: HOXD10 silencing suppresses human fibroblast-like synoviocyte migration in rheumatoid arthritis via downregulation of the p38/JNK pathway. Exp Ther Med 2018, 16(3):16211628.

35. Inoue T, Hammaker D, Boyle DL, Firestein GS: Regulation of p38 MAPK by MAPK kinases 3 and 6 in fibroblast-like synoviocytes. J Immunol 2005, 174(7):4301-4306.

36. Inoue T, Hammaker D, Boyle DL, Firestein GS: Regulation of p38 MAPK by MAPK Kinases 3 and 6 in Fibroblast-Like Synoviocytes. The Journal of Immunology 2005, 174(7):4301-4306.

37. Avdi NJ, Nick JA, Whitlock BB, Billstrom MA, Henson PM, Johnson GL, Worthen GS: Tumor necrosis factor-alpha activation of the c-Jun N-terminal kinase pathway in human neutrophils. Integrin involvement in a pathway leading from cytoplasmic tyrosine kinases apoptosis. J Biol Chem 2001, 276(3):2189-2199.

38. Liao C, Hsu J, Kim Y, Hu DQ, Xu DG, Zhang J, Pashine A, Menke J, Whittard T, Romero N et al: Selective inhibition of spleen tyrosine kinase (SYK) with a novel orally bioavailable small molecule 
inhibitor, R09021, impinges on various innate and adaptive immune responses: implications for SYK inhibitors in autoimmune disease therapy. Arthritis Research \& Therapy 2013, 15(5).

39. Ahmed S, Riegsecker S, Beamer M, Rahman A, Bellini JV, Bhansali P, Tillekeratne LM: Largazole, a class I histone deacetylase inhibitor, enhances TNF-alpha-induced ICAM-1 and VCAM-1 expression in rheumatoid arthritis synovial fibroblasts. Toxicol Appl Pharmacol 2013, 270(2):87-96.

40. Yin HS, Li YJ, Jiang ZA, Liu SY, Guo BY, Wang T: Nicotine-induced ICAM-1 and VCAM-1 expression in mouse cardiac vascular endothelial cell via p38 MAPK signaling pathway. Anal Quant Cytopathol Histpathol 2014, 36(5):258-262.

\section{Figures}

A

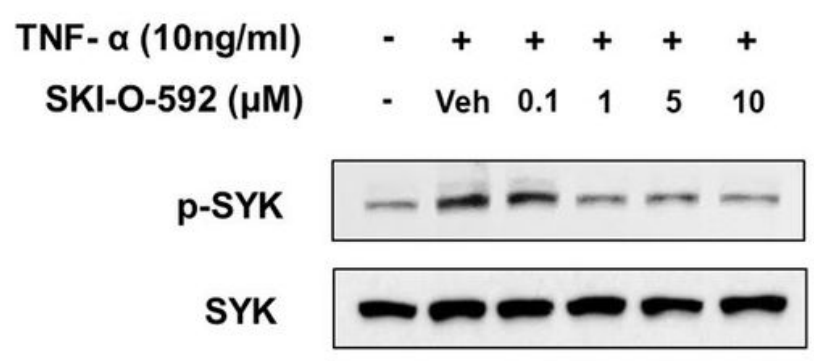

B

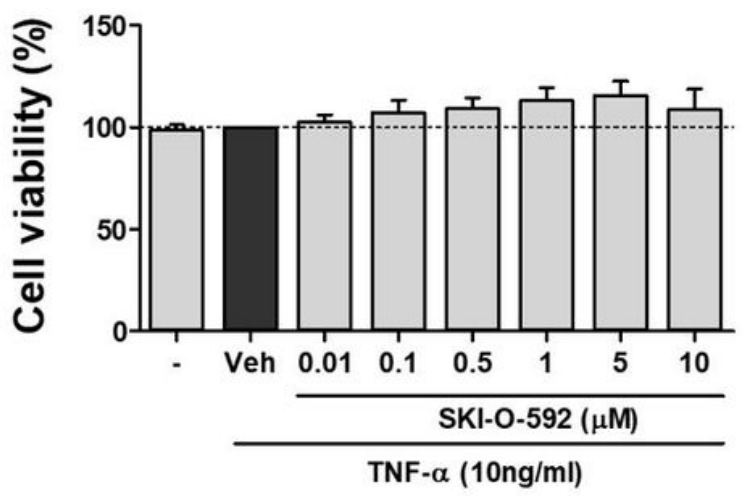

Figure 1

SKI-0-592 reduces SYK phosphorylation in RA FLS. RA FLS $(n=3)$ were pretreated with vehicle $(0.1 \%$ DMSO) or SKI-O-592 for 1 hour and then stimulated with $10 \mathrm{ng} / \mathrm{ml} \mathrm{TNF-a} \mathrm{for} 15$ minutes. Total proteins were extracted and evaluated by Western blot analysis. A representative blot of independent experiments is shown. (A) TNF-a-induced phospho-SYK expression was significantly decreased by SKI-0-592. (B) SKI0-592 had no significant effect on the viability of RA FLS $(n=6)$. Data shown are representative of experiments from different RA patients and presented as the mean \pm SEM.

A

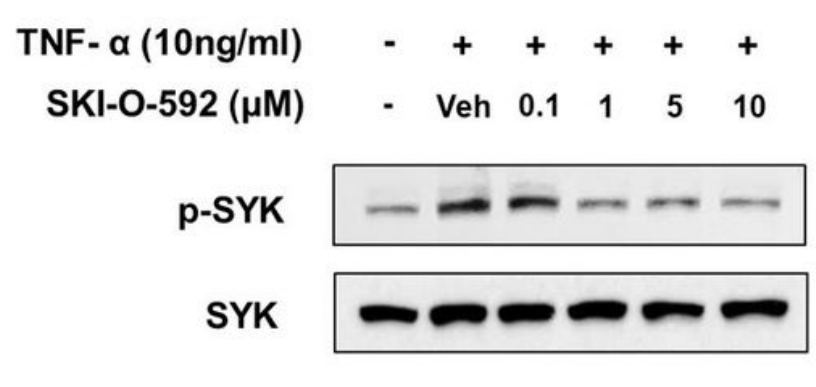

B

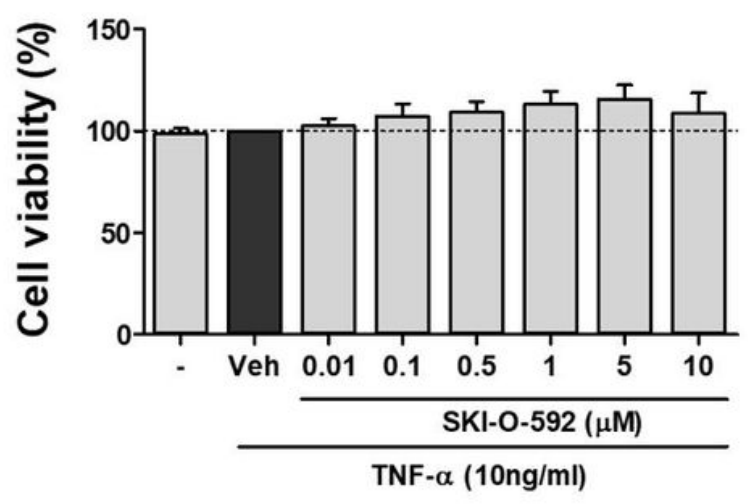


Figure 1

SKI-0-592 reduces SYK phosphorylation in RA FLS. RA FLS $(\mathrm{n}=3)$ were pretreated with vehicle $(0.1 \%$ DMSO) or SKI-0-592 for 1 hour and then stimulated with $10 \mathrm{ng} / \mathrm{ml}$ TNF-a for 15 minutes. Total proteins were extracted and evaluated by Western blot analysis. A representative blot of independent experiments is shown. (A) TNF-a-induced phospho-SYK expression was significantly decreased by SKI-O-592. (B) SKI$0-592$ had no significant effect on the viability of RA FLS $(n=6)$. Data shown are representative of experiments from different RA patients and presented as the mean \pm SEM.

A
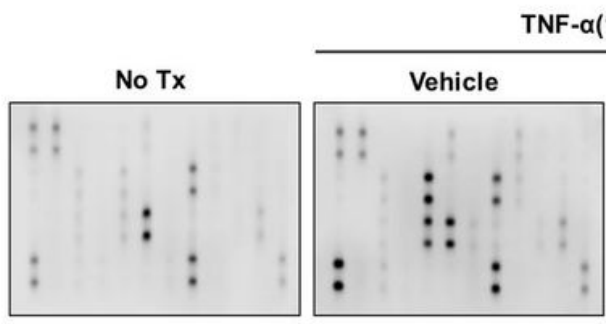

NF- $\alpha(10 \mathrm{ng} / \mathrm{ml})$

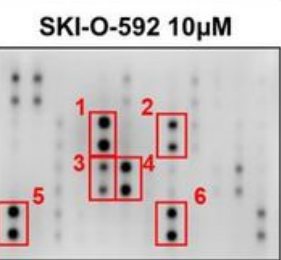

1. IL-6

2. IL-8

3. $\mathrm{CXCL10}$

4. MCP-1

5. RANTES

6. TIMP-2
B

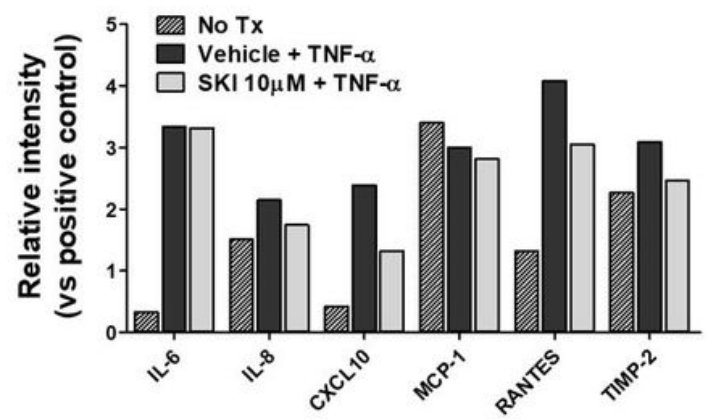

C

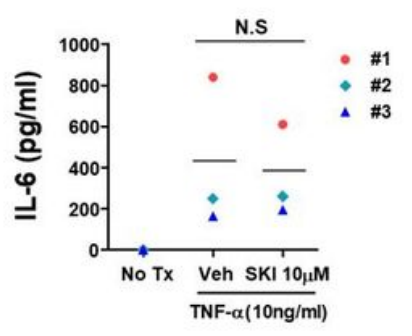

D

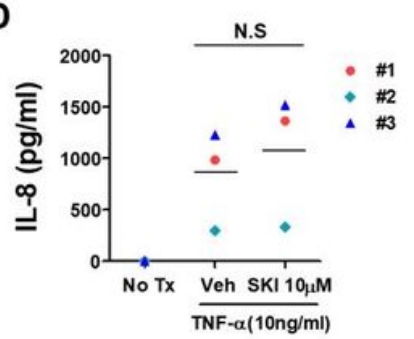

E

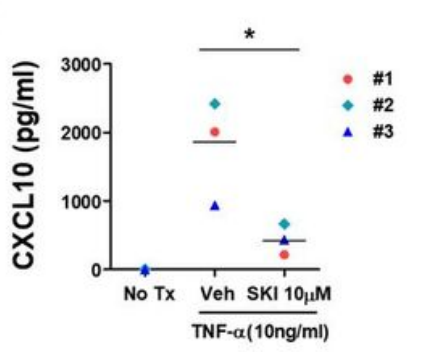

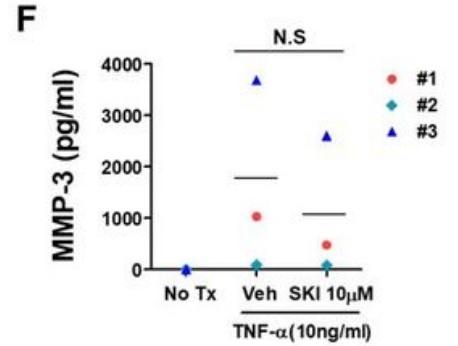

\section{Figure 2}

SKI-0-592 affects the production of cytokines, chemokine, and MMP in RA FLS stimulated by TNF- $a$. RA FLS $(n=3)$ were pretreated with vehicle (0.1\% DMSO) or SKI-0-592 for 1 hour, then stimulated with TNF-a for 48 hours. (A) Representative image of antibody array results using culture supernatant. (B) The dot expression intensity of each molecule was quantified by densitometry, and the production of IL-8, CXCL10, MCP-1, RANTES, and TIMP-2 were decreased by SKI-0-592 compared to the vehicle-treated group, except for IL-6. SKI-0-592 did not affect the secretion of (C) IL-6, (D) IL-8, and (F) MMP-3 but decreased secretion of (E) CXCL10. Data are presented as the mean \pm SEM. * $p<0.05$ as compared with the vehicle group using Student's t-test. The groups not treated with any drug or vehicle are indicated as 'No Tx'. 
A
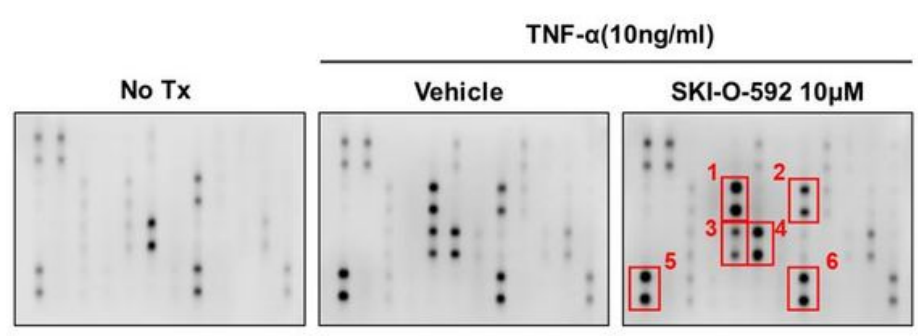

1. IL-6

2. IL-8

3. CXCL10

4. $M C P-1$

5. RANTES

6. TIMP-2
B

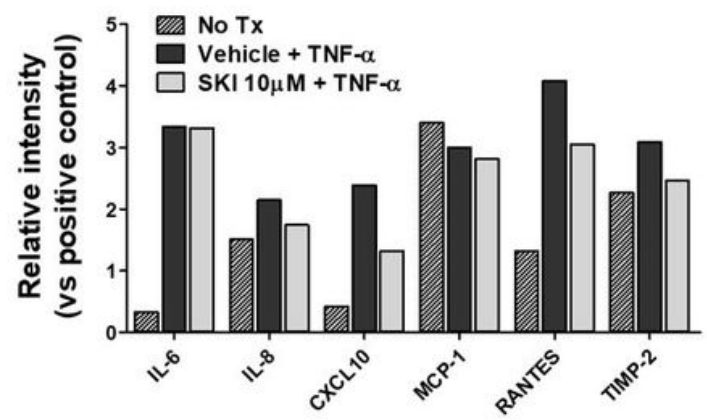

C

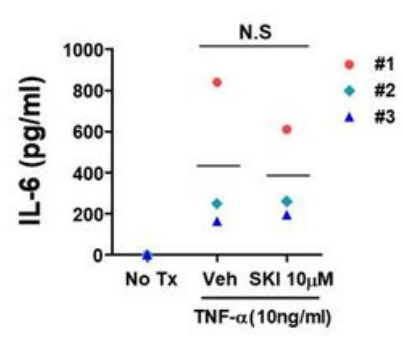

D

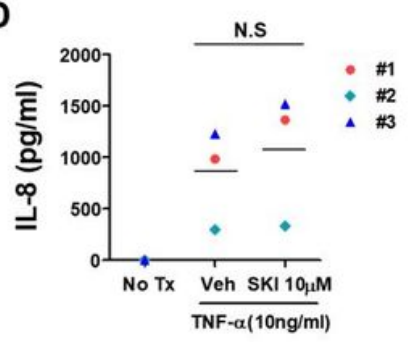

E

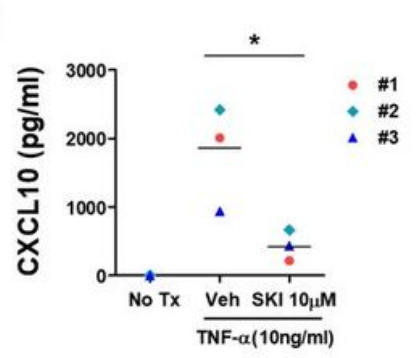

$\mathbf{F}$

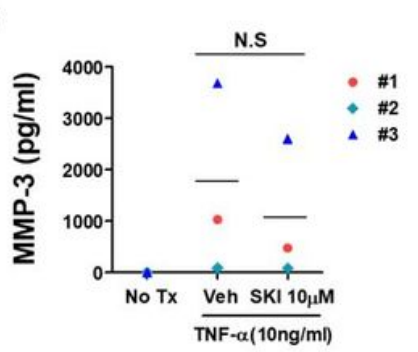

Figure 2

SKI-0-592 affects the production of cytokines, chemokine, and MMP in RA FLS stimulated by TNF-a. RA FLS $(n=3)$ were pretreated with vehicle (0.1\% DMSO) or SKI-0-592 for 1 hour, then stimulated with TNF-a for 48 hours. (A) Representative image of antibody array results using culture supernatant. (B) The dot expression intensity of each molecule was quantified by densitometry, and the production of IL-8, CXCL10, MCP-1, RANTES, and TIMP-2 were decreased by SKI-0-592 compared to the vehicle-treated group, except for IL-6. SKI-0-592 did not affect the secretion of (C) IL-6, (D) IL-8, and (F) MMP-3 but decreased secretion of (E) CXCL10. Data are presented as the mean \pm SEM. * $p<0.05$ as compared with the vehicle group using Student's t-test. The groups not treated with any drug or vehicle are indicated as 'No Tx'. 
A

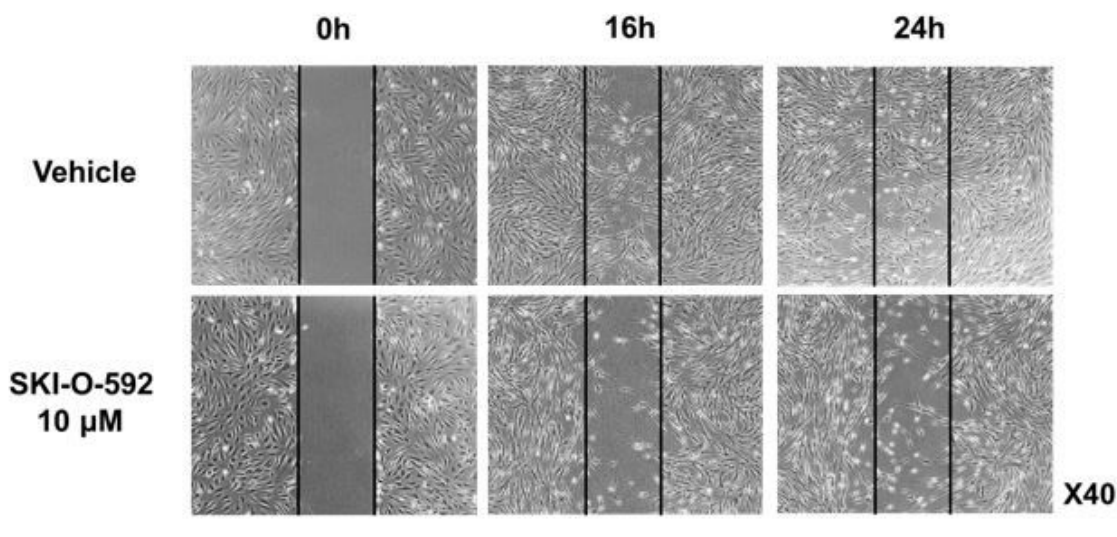

B

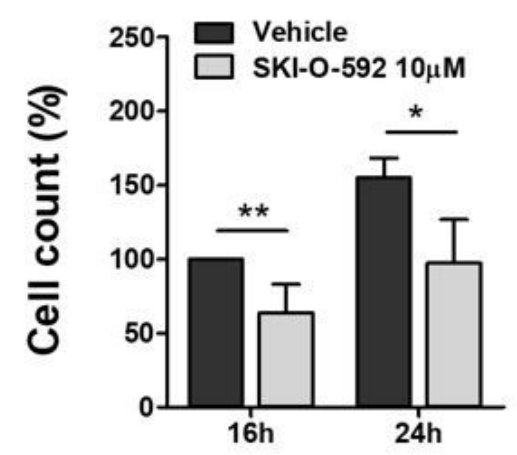

C

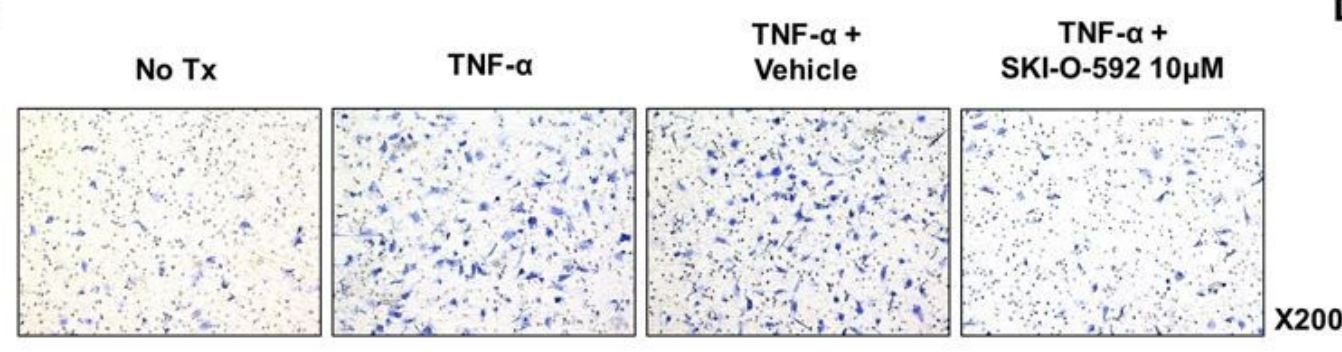

D

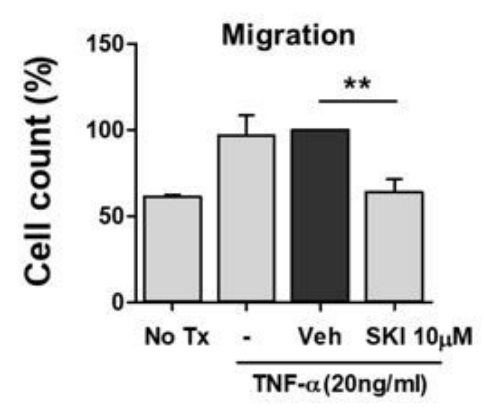

E

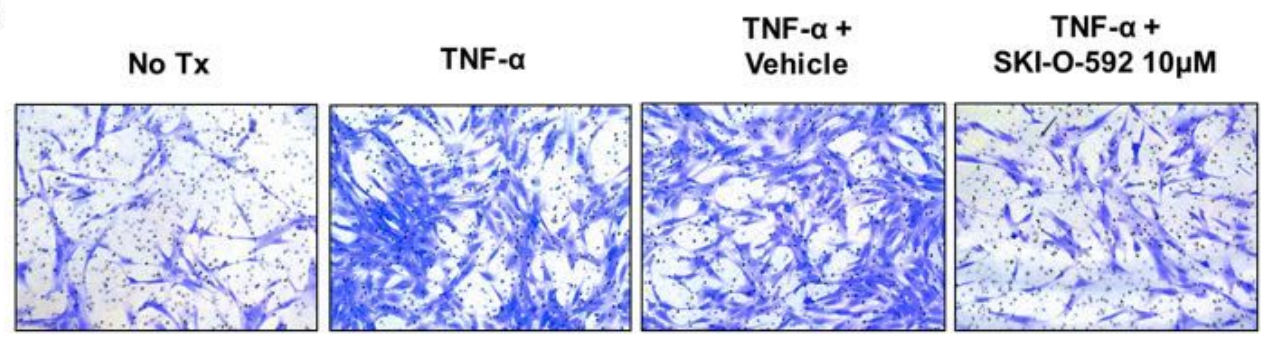

$\mathrm{X} 200$

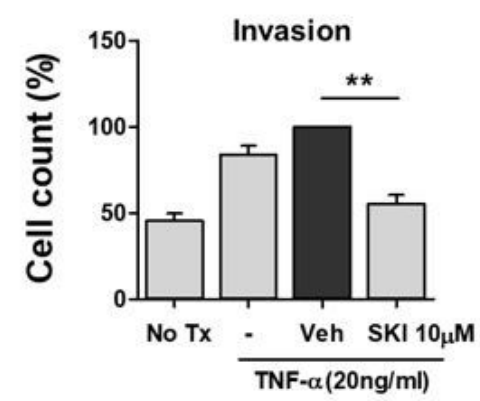

Figure 3

SKI-0-592 suppresses cell migration and invasion ability of RA FLS. Wound healing assay was performed to evaluate the migration capability of cells. (A) Cell migration was decreased in SKI-0-592-treated RA FLS ( $n=3)$, at both $16 \mathrm{hr}$ and $24 \mathrm{hr}$ after scratch wound. (B) Numbers of cells migrating beyond the black line on microscopy images were counted. Representative images of (C) migrated and (E) invaded cells stained with crystal violet are shown. (D) Cell migration and $(F)$ invasion was evaluated by cell counting. Data shown are representative of experiments from four different RA patients and presented as the mean $\pm S E M . * p<0.05,{ }^{*} p<0.01$, as compared with vehicle group using Student's t-test. The groups not treated with any drug or vehicle are indicated as 'No Tx'. 
A

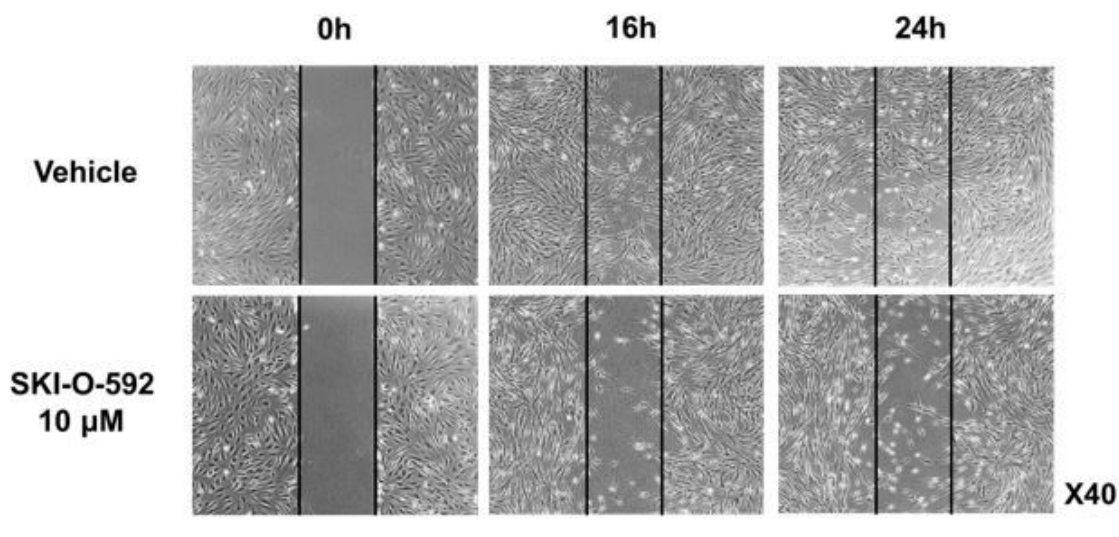

B

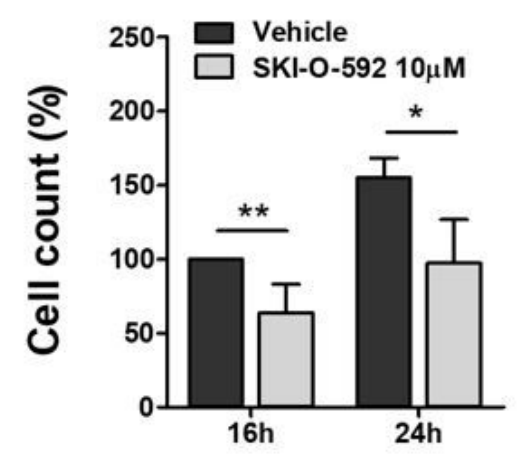

C

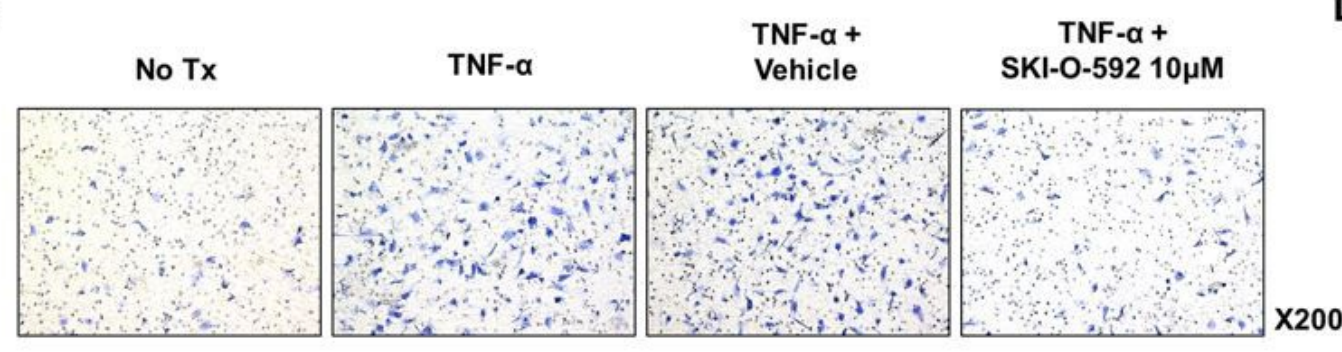

D

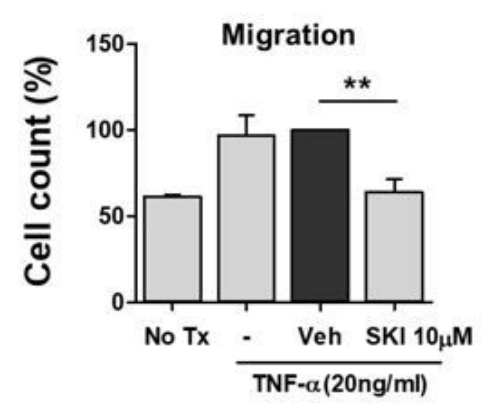

E

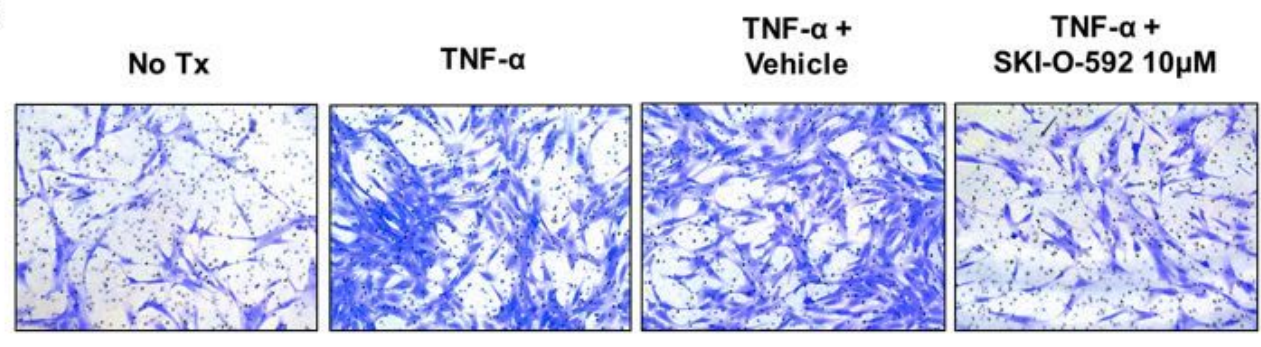

$\mathrm{X} 200$

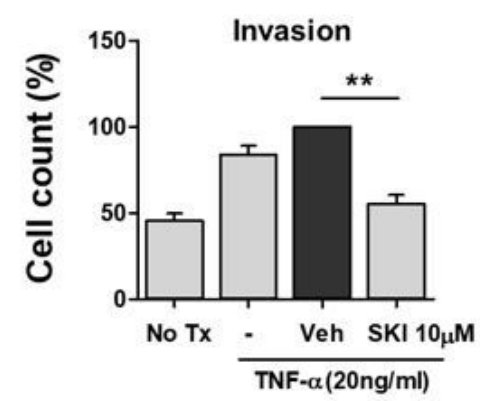

Figure 3

SKI-0-592 suppresses cell migration and invasion ability of RA FLS. Wound healing assay was performed to evaluate the migration capability of cells. (A) Cell migration was decreased in SKI-0-592-treated RA FLS ( $n=3)$, at both $16 \mathrm{hr}$ and $24 \mathrm{hr}$ after scratch wound. (B) Numbers of cells migrating beyond the black line on microscopy images were counted. Representative images of (C) migrated and (E) invaded cells stained with crystal violet are shown. (D) Cell migration and $(F)$ invasion was evaluated by cell counting. Data shown are representative of experiments from four different RA patients and presented as the mean $\pm S E M . * p<0.05,{ }^{*} p<0.01$, as compared with vehicle group using Student's t-test. The groups not treated with any drug or vehicle are indicated as 'No Tx'. 
A
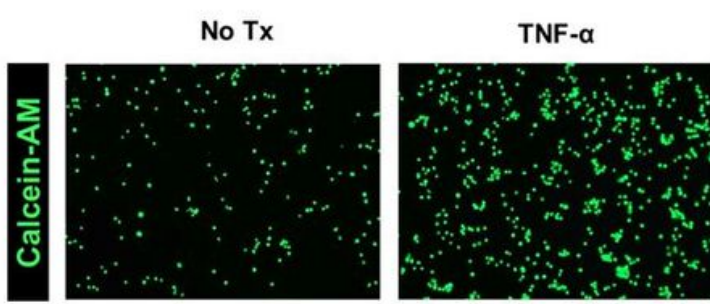

C

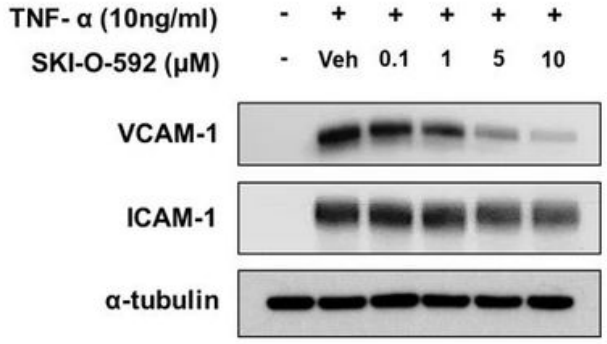

B

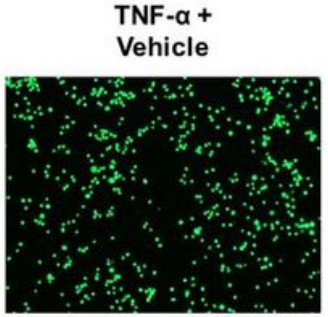

TNF- $\alpha+$ SKI-O-592 10 $\mu \mathrm{M}$

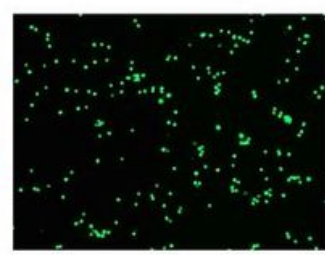

$\mathrm{X} 100$

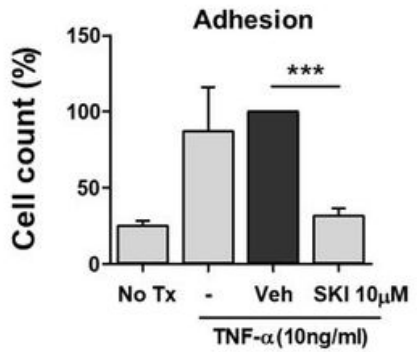

D

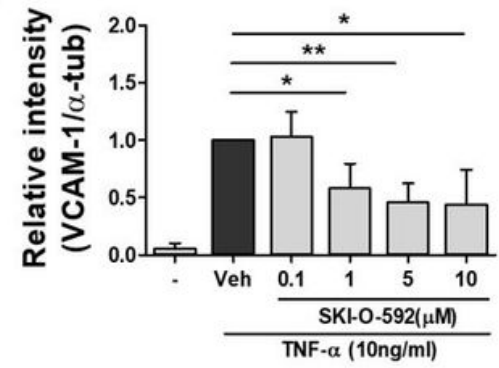

E

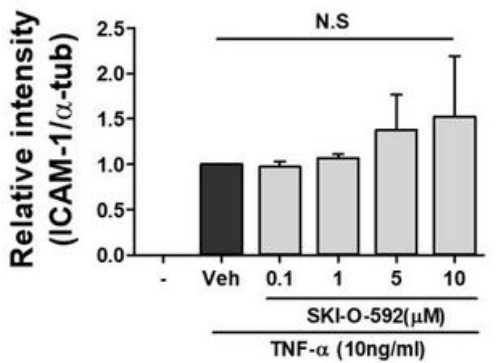

\section{Figure 4}

SKI-0-592 decreases cell adhesion and downregulates the expression of adhesion molecules in RA FLS. Cell adhesion assay was performed to investigate monocyte adhesion to SKI-0-592-treated RA FLS. (A, B) Adhesion of calcein-labeled THP-1 to RA FLS ( $n=3)$ was evaluated by counting cells with positive fluorescence. (C-E) The expression levels of VCAM-1 and ICAM-1 were detected by Western blot. Data shown are representative of experiments from three different RA patients, and data are presented as the mean \pm SEM. * $p<0.05, * \star p<0.01, * \star * p<0.001$ as compared with the vehicle group using Student's ttest. The groups not treated with any drug or vehicle are indicated as 'No Tx'. 
A
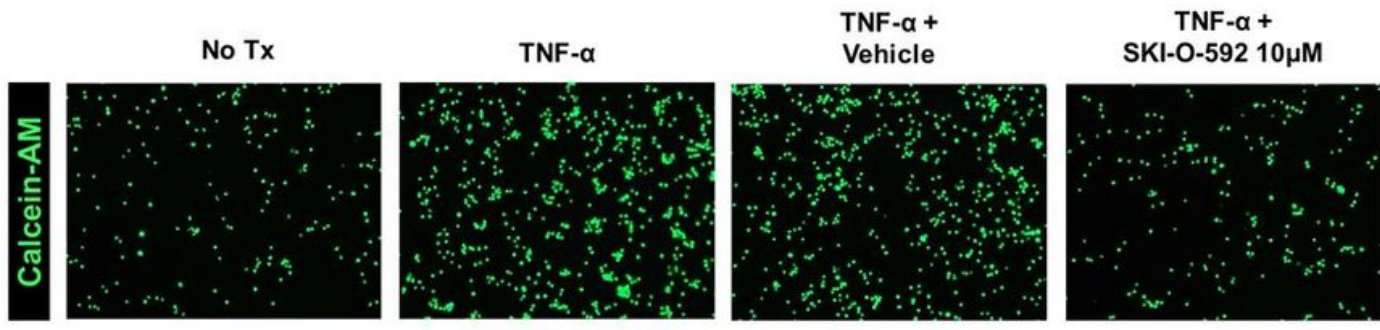

$\mathrm{X} 100$

B

TNF- $\alpha+$

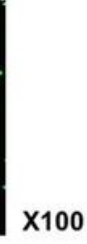

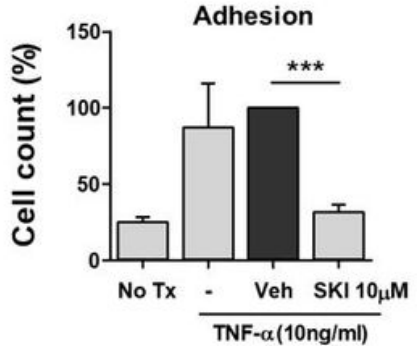

E
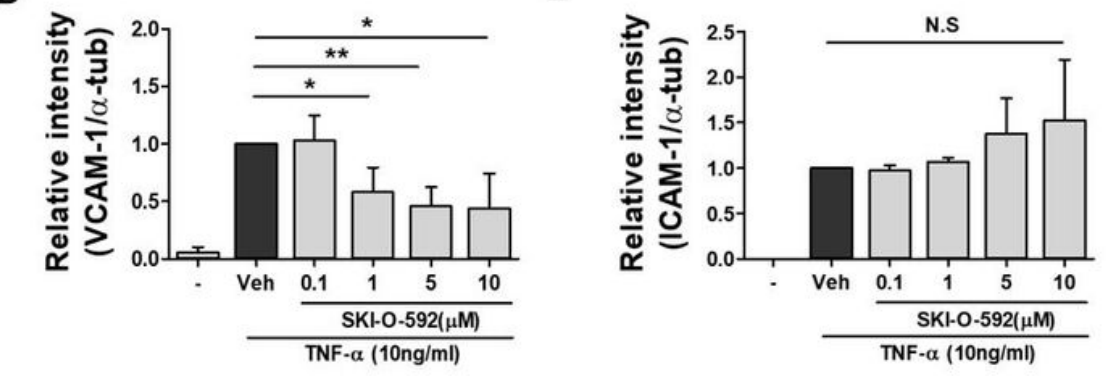

Figure 4

SKI-0-592 decreases cell adhesion and downregulates the expression of adhesion molecules in RA FLS. Cell adhesion assay was performed to investigate monocyte adhesion to SKI-0-592-treated RA FLS. (A, B) Adhesion of calcein-labeled THP-1 to RA FLS ( $n=3)$ was evaluated by counting cells with positive fluorescence. (C-E) The expression levels of VCAM-1 and ICAM-1 were detected by Western blot. Data shown are representative of experiments from three different RA patients, and data are presented as the mean \pm SEM. * $p<0.05,{ }^{* *} p<0.01,{ }^{* \star *} p<0.001$ as compared with the vehicle group using Student's ttest. The groups not treated with any drug or vehicle are indicated as 'No Tx'. 
A

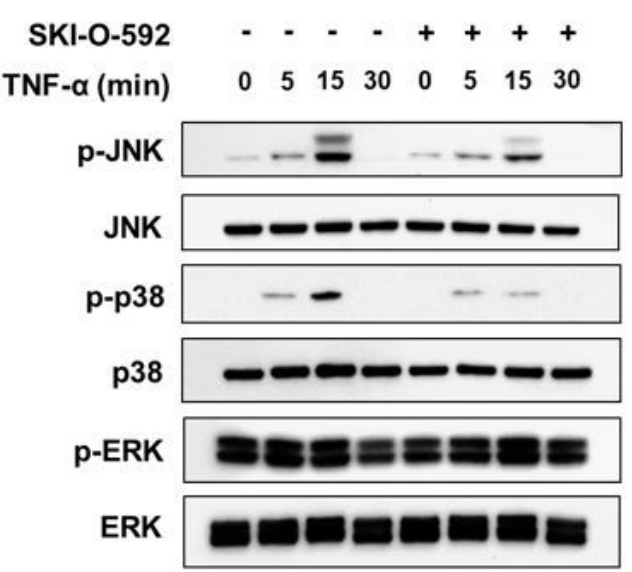

C

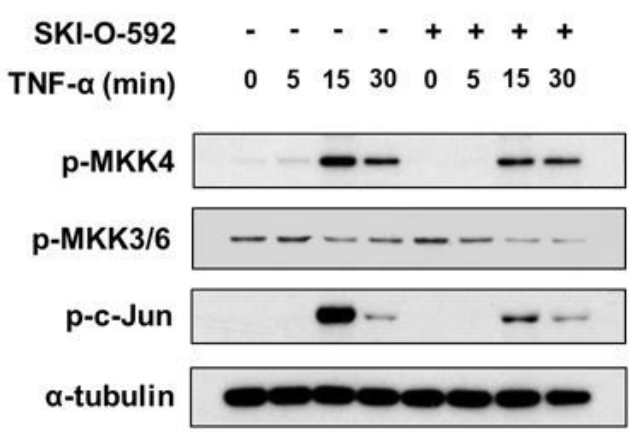

B

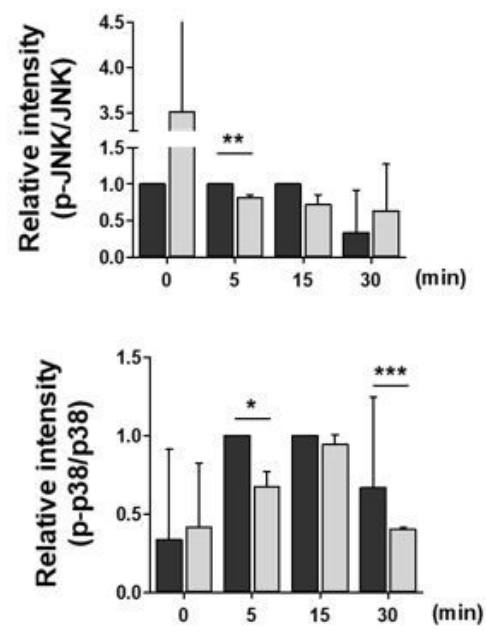

Vehicle

SKI-O-592
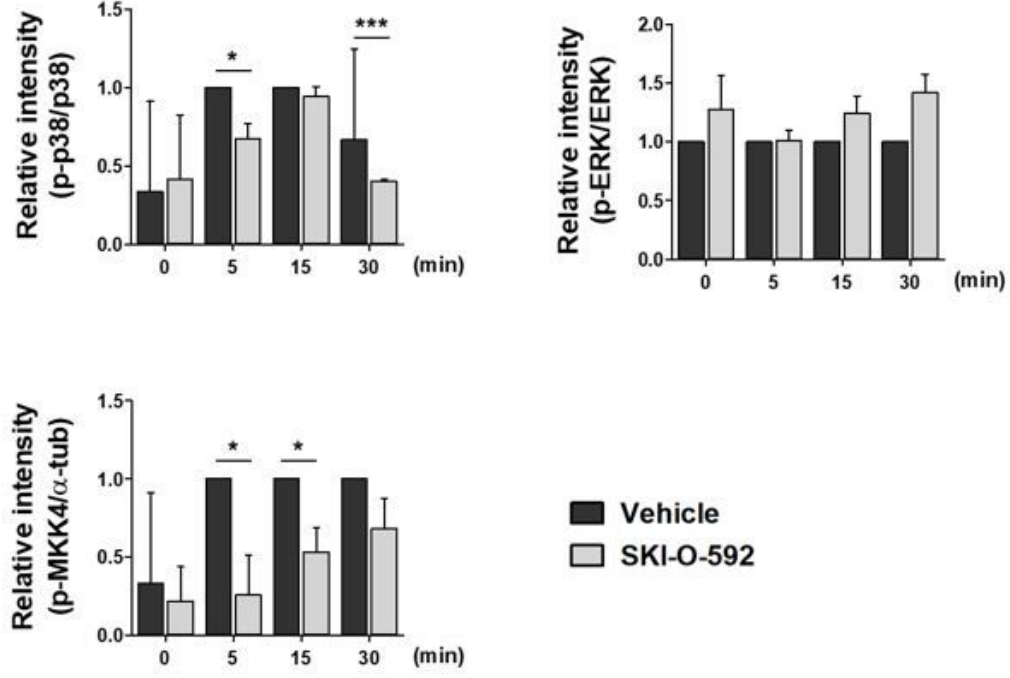

Vehicle

SKI-O-592
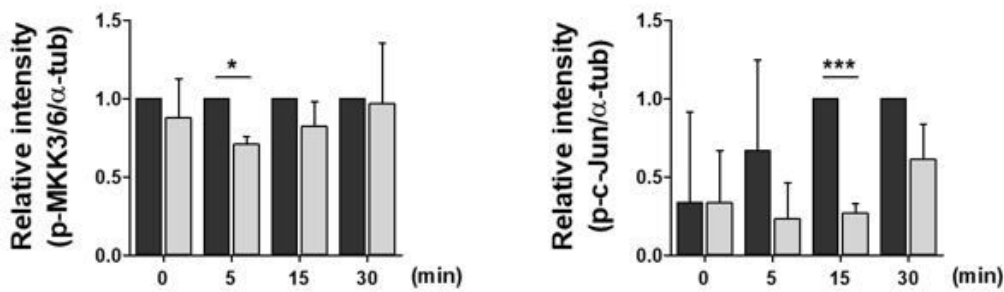

\section{Figure 5}

Effect of SKI-0-592 on TNF-a induced MAPKs phosphorylation. RA FLS $(n=3)$ were treated with DMSO or $10 \mu \mathrm{M}$ SKI-0-592. One hr later, cells were stimulated with $10 \mathrm{ng} / \mathrm{ml}$ TNF-a for 5 to $30 \mathrm{~min}$. Total proteins were extracted and evaluated by Western blot. Data are shown as the ratio of band intensity for the phosphorylated MAPK to total MAPK (e.g., phospho-JNK/JNK) or a-tubulin. (A, B) SKI-0-592 decreased TNF-a-induced phosphorylation of JNK and p38 (Figure 5A, B). However, ERK phosphorylation was not affected. (C, D) Decreases of phospho-MKK4 and MKK3/6 by SKI-0-592 were observed after TNF-a stimulation. TNF-a-induced phospho-C-Jun expression was significantly decreased by SKI-0-592. A representative blot of independent experiments is shown. Data are presented as the mean \pm SEM. * $p<$ $0.05,{ }^{\star \star} p<0.01, \star \star \star p<0.001$ as compared with the vehicle group using Student's t-test. 
A

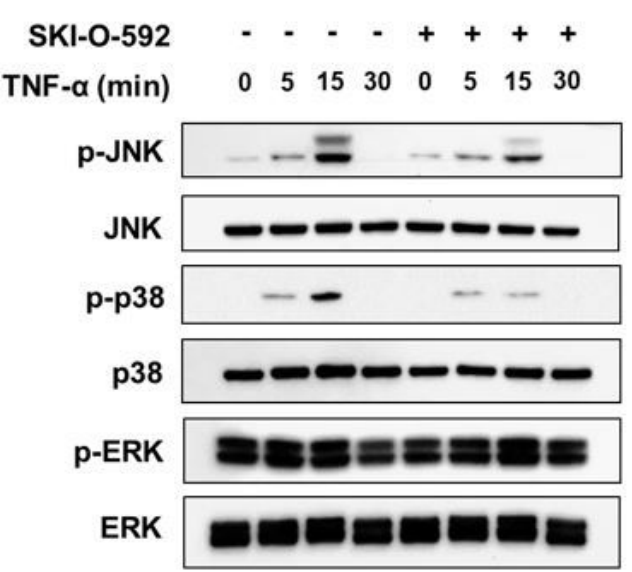

C

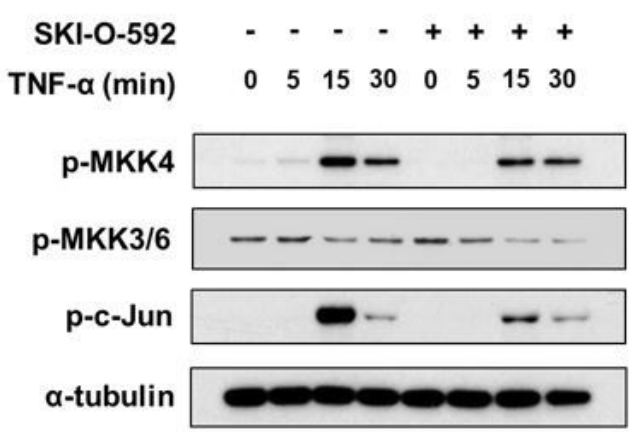

B

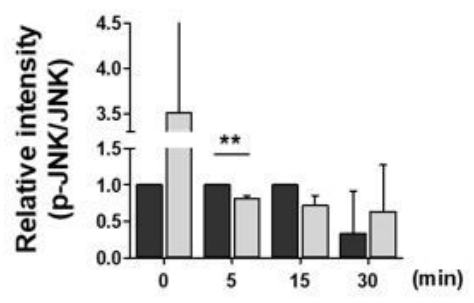

Vehicle

SKI-O-592
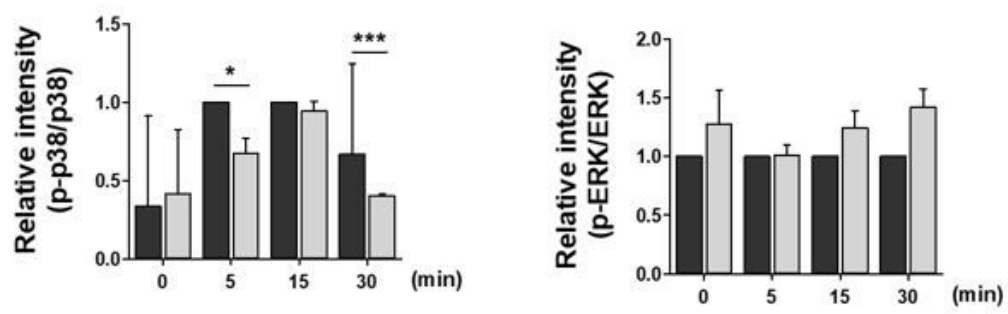

D

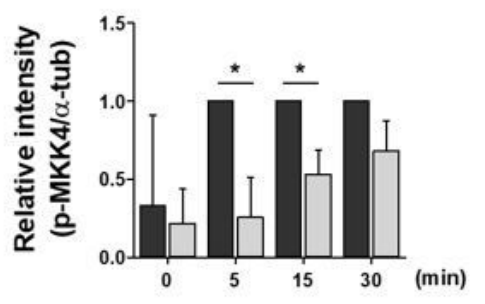

Vehicle

SKI-O-592
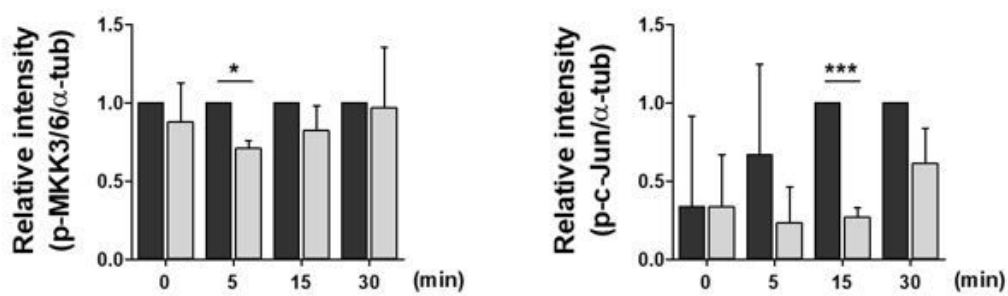

\section{Figure 5}

Effect of SKI-0-592 on TNF-a induced MAPKs phosphorylation. RA FLS $(n=3)$ were treated with DMSO or $10 \mu \mathrm{M}$ SKI-0-592. One hr later, cells were stimulated with $10 \mathrm{ng} / \mathrm{ml} \mathrm{TNF-a} \mathrm{for} 5$ to $30 \mathrm{~min}$. Total proteins were extracted and evaluated by Western blot. Data are shown as the ratio of band intensity for the phosphorylated MAPK to total MAPK (e.g., phospho-JNK/JNK) or a-tubulin. (A, B) SKI-0-592 decreased TNF-a-induced phosphorylation of JNK and p38 (Figure 5A, B). However, ERK phosphorylation was not affected. (C, D) Decreases of phospho-MKK4 and MKK3/ 6 by SKI-0-592 were observed after TNF-a stimulation. TNF-a-induced phospho-C-Jun expression was significantly decreased by SKI-0-592. A representative blot of independent experiments is shown. Data are presented as the mean \pm SEM. * $p<$ $0.05,{ }^{\star \star} p<0.01, * \star \star p<0.001$ as compared with the vehicle group using Student's t-test. 
A

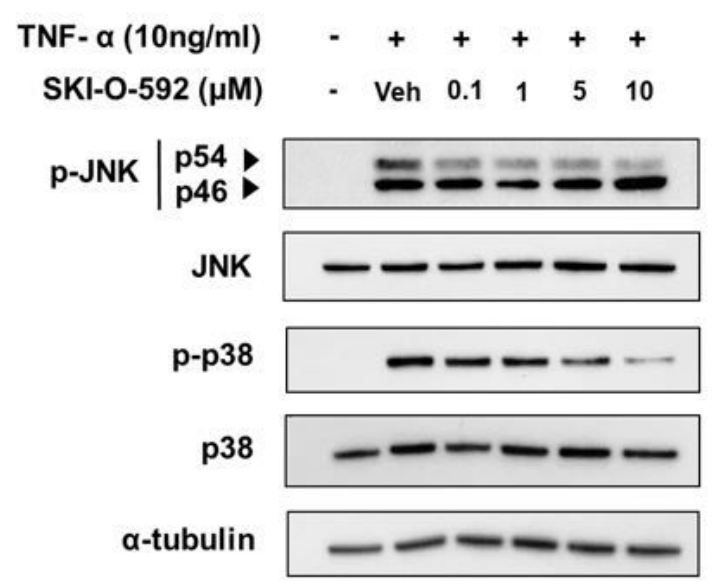

B
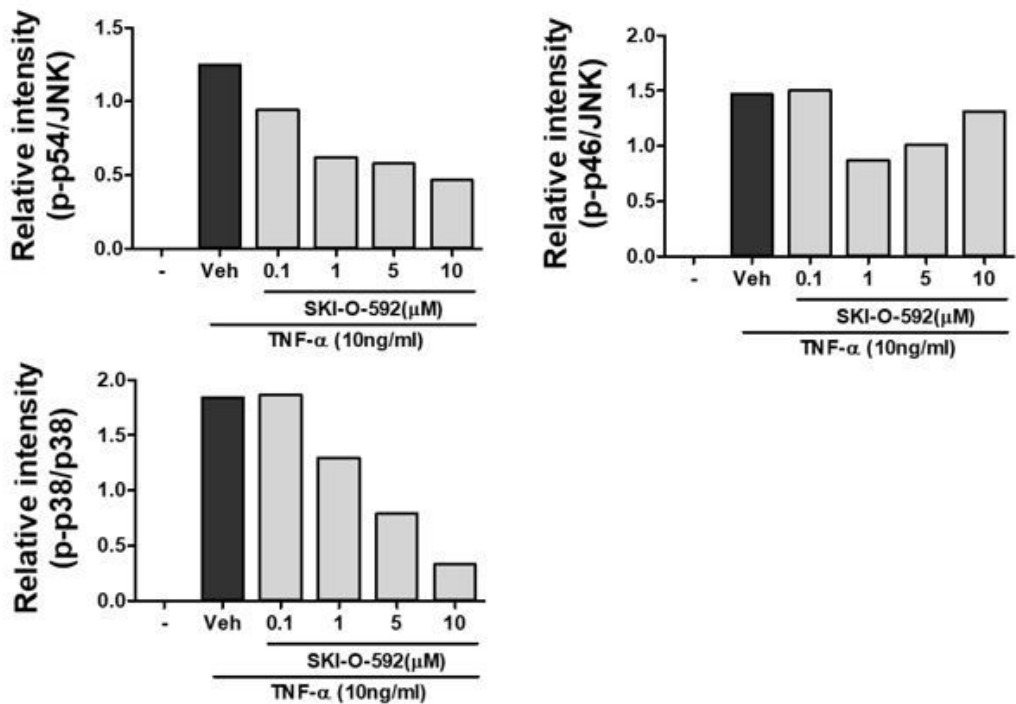

Figure 6

SKI-0-592 dose-dependently downregulates the phosphorylation of JNK and p38. RA FLS $(n=1)$ were treated with DMSO or $10 \mu \mathrm{M} \mathrm{SKI-0-592.} \mathrm{(A)} \mathrm{One} \mathrm{hr} \mathrm{later,} \mathrm{cells} \mathrm{were} \mathrm{stimulated} \mathrm{with} 10 \mathrm{ng} / \mathrm{ml} \mathrm{TNF-a} \mathrm{for} 15$ min. Total proteins were extracted and evaluated by Western blot analysis for JNK, p38, and their phosphorylation. Data are shown as the ratio of band intensity for the phosphorylated to total protein or a-tubulin. (B) Phosphorylation of p54 JNK and p38 was dose-dependently decreased by SKI-0-592. A representative blot of independent experiments is shown. Data are presented as the mean \pm SEM.

A

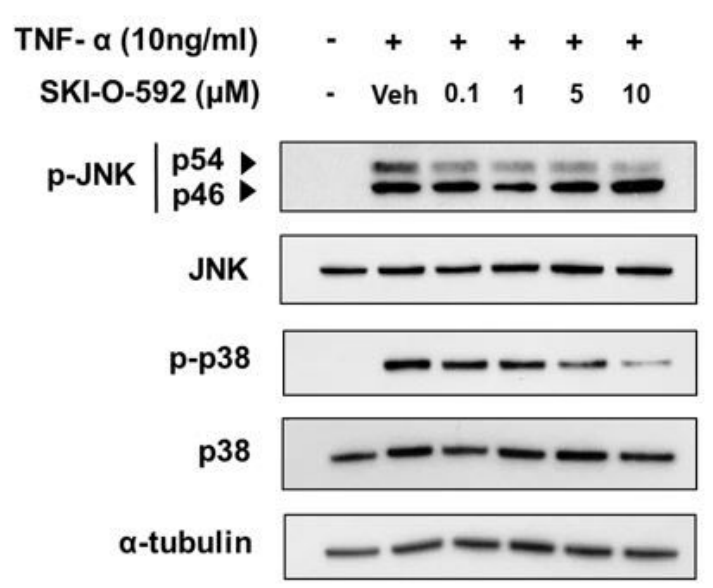

B
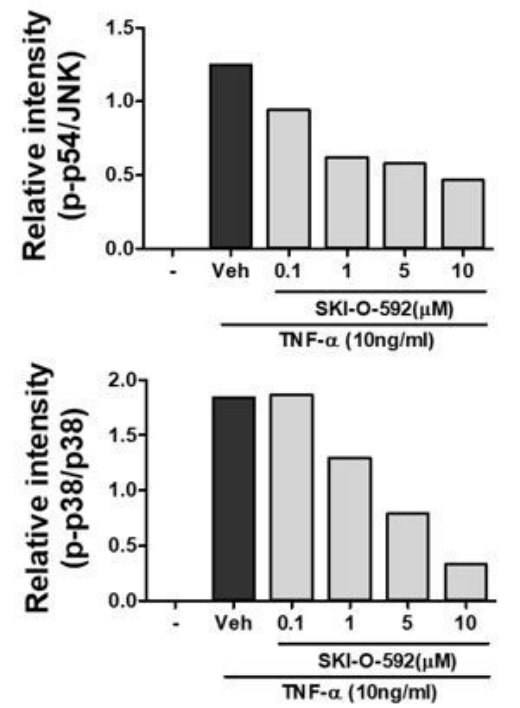

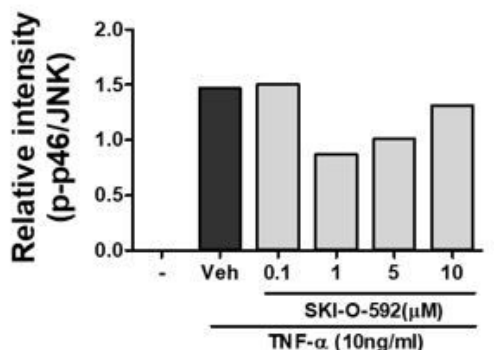

TNF- $\alpha$ (10ng/ml)

Figure 6 
SKI-0-592 dose-dependently downregulates the phosphorylation of JNK and p38. RA FLS $(n=1)$ were treated with DMSO or $10 \mu \mathrm{M}$ SKI-0-592. (A) One hr later, cells were stimulated with $10 \mathrm{ng} / \mathrm{ml}$ TNF-a for 15 min. Total proteins were extracted and evaluated by Western blot analysis for JNK, p38, and their phosphorylation. Data are shown as the ratio of band intensity for the phosphorylated to total protein or a-tubulin. (B) Phosphorylation of p54 JNK and p38 was dose-dependently decreased by SKI-0-592. A representative blot of independent experiments is shown. Data are presented as the mean \pm SEM.

A

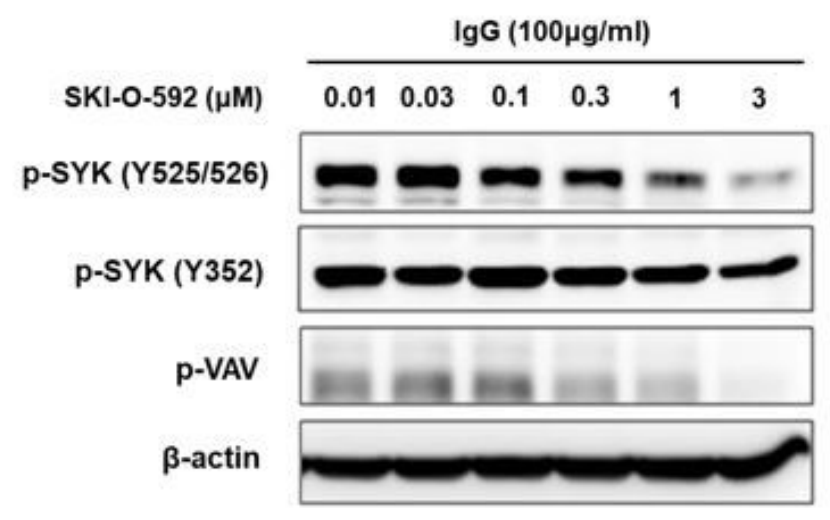

B

C
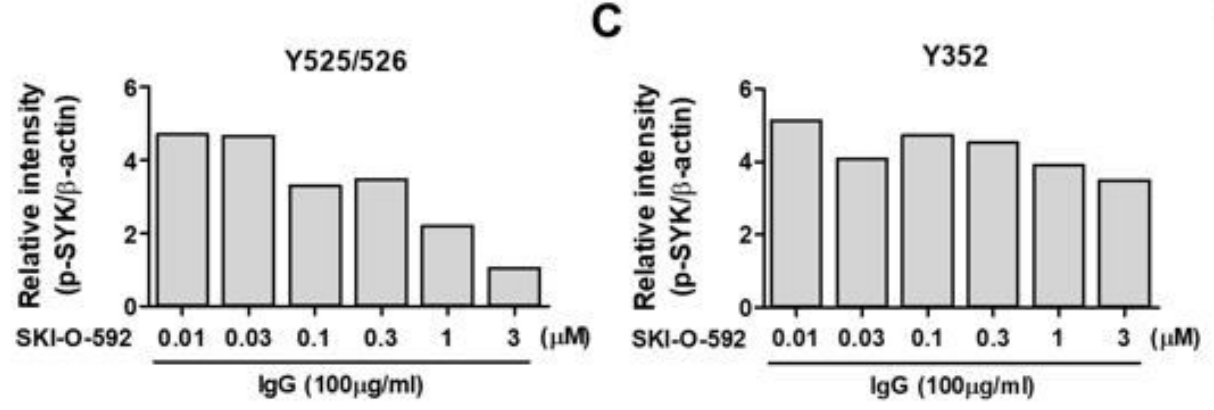

D

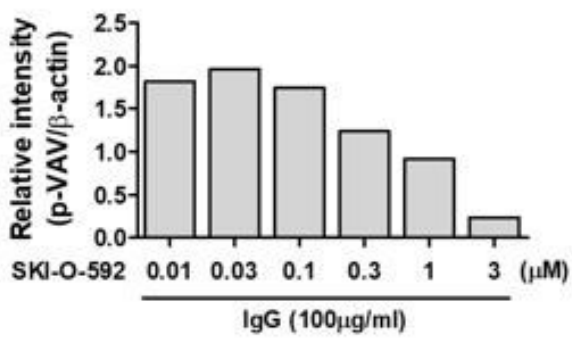

E

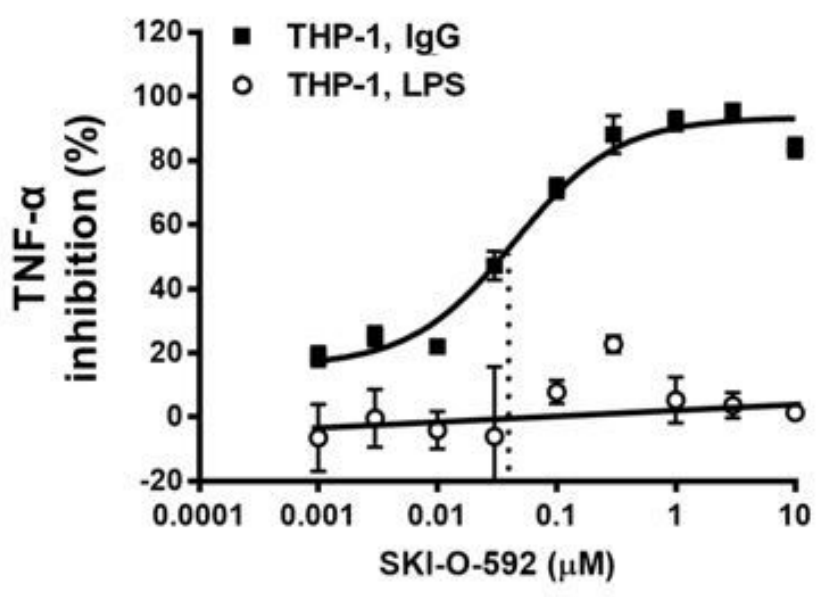

F

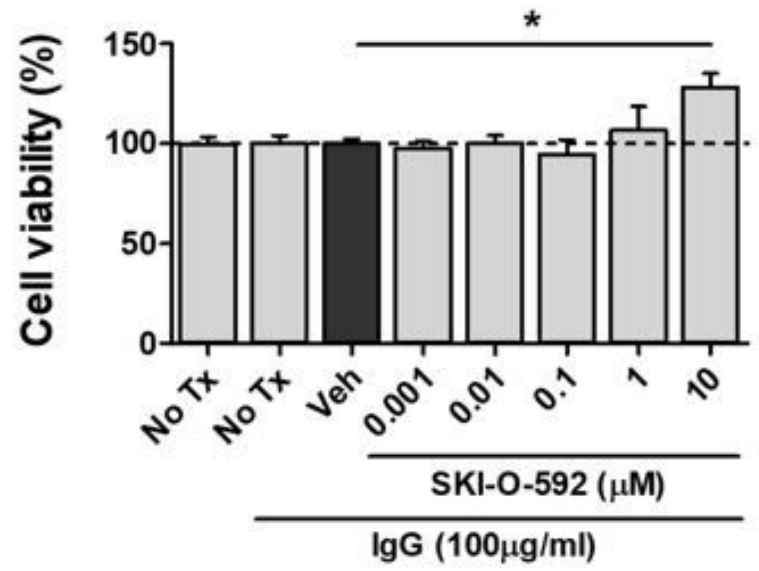

Figure 7 
Inhibitory effect of SKI-0-592 on THP-1 cells. To evaluate the inhibition of FcyR-mediated SYK pathways in monocytes, THP-1 cells were plated at $1 \times 105$ cells/well into human IgG precoated 96 -well plates and SKI-0-592 was added serially from 0.001 to $10 \mu \mathrm{M}$. (A) Immunoblot of the whole cell lysate showed inhibition of the phosphorylation levels of various sites in SYK-dependent pathway kinases such as (B) Y525/526 and (C) Y352 or (D) VAV on THP-1. (E) TNF-a in the culture supernatant was decreased dosedependently by SKI-0-592 treatment in IgG treated group compared to the LPS-treated group. (F) THP-1 cells were subjected to a CCK-8 assay to evaluate the cytotoxic effects of compounds. There was no significant change in cell viability. The data represent a mean $\pm S D$ of duplicate cultures. ${ }^{*} p<0.05$ as compared with the vehicle group using Student's t-test. Groups not treated with any drug or vehicle are indicated as 'No Tx'.

A

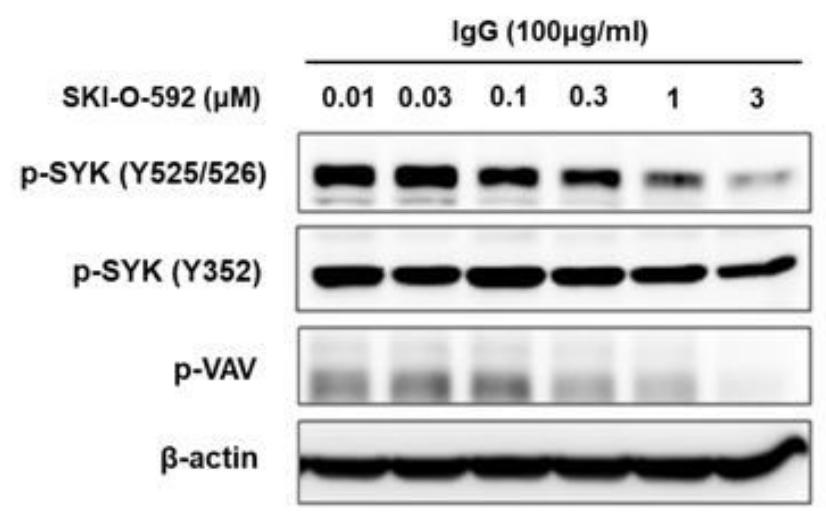

B

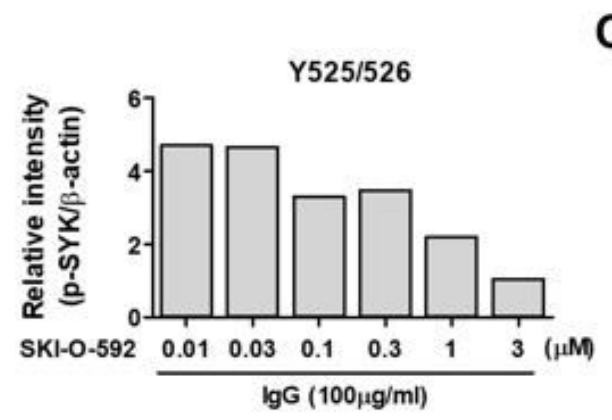

C

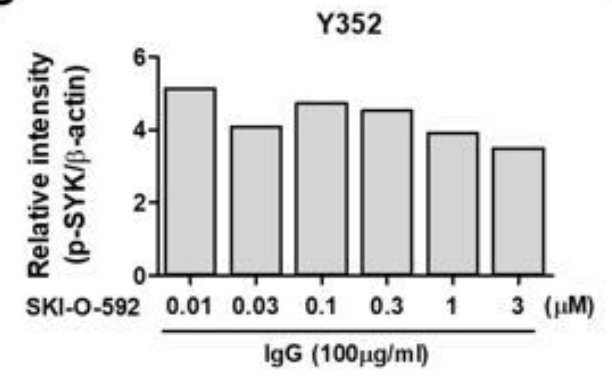

D

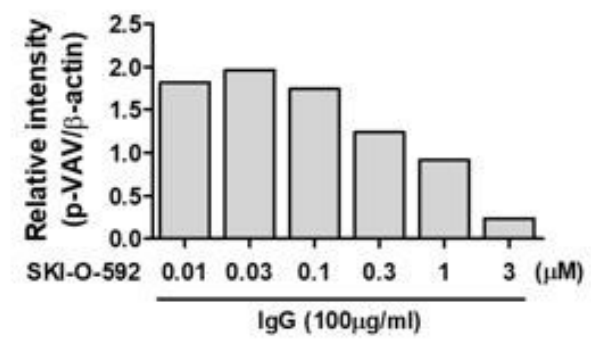

E

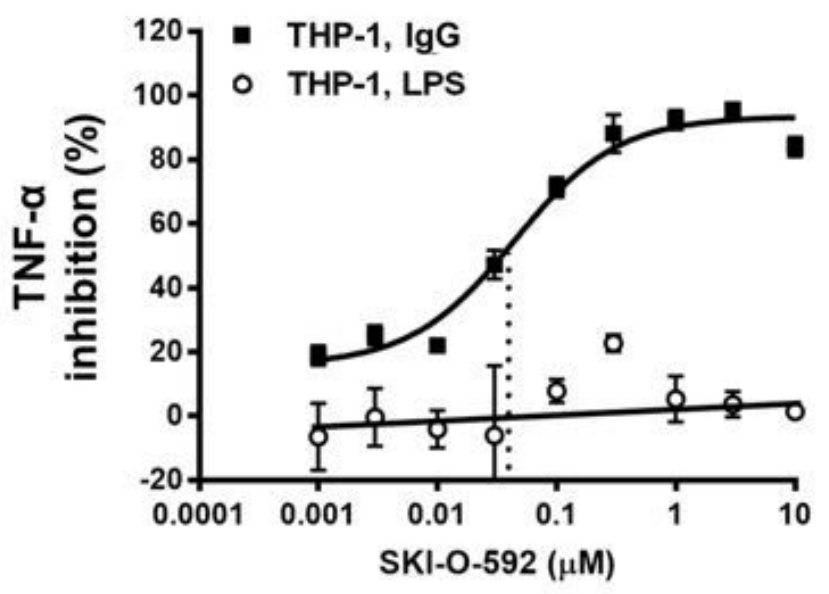

F

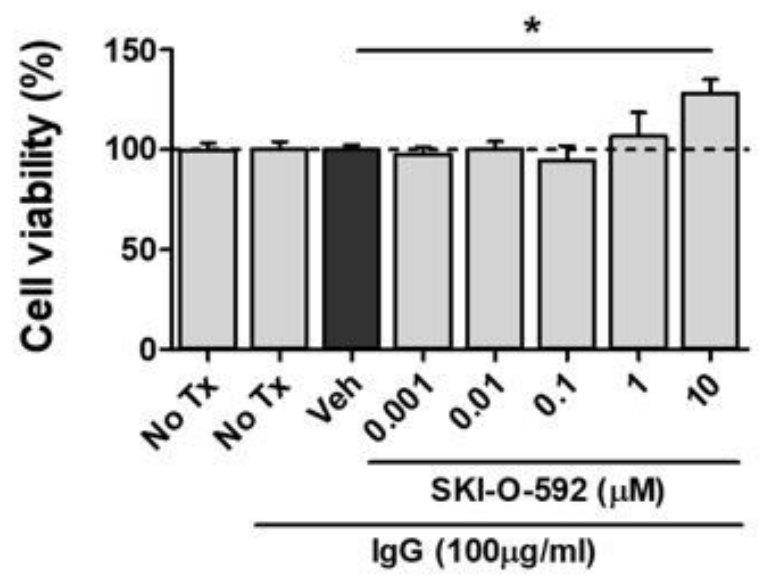




\section{Figure 7}

Inhibitory effect of SKI-0-592 on THP-1 cells. To evaluate the inhibition of FcyR-mediated SYK pathways in monocytes, THP-1 cells were plated at $1 \times 105$ cells/well into human IgG precoated 96 -well plates and SKI-0-592 was added serially from 0.001 to $10 \mu \mathrm{M}$. (A) Immunoblot of the whole cell lysate showed inhibition of the phosphorylation levels of various sites in SYK-dependent pathway kinases such as (B) Y525/526 and (C) Y352 or (D) VAV on THP-1. (E) TNF-a in the culture supernatant was decreased dosedependently by SKI-O-592 treatment in IgG treated group compared to the LPS-treated group. (F) THP-1 cells were subjected to a CCK-8 assay to evaluate the cytotoxic effects of compounds. There was no significant change in cell viability. The data represent a mean $\pm S D$ of duplicate cultures. ${ }^{*} p<0.05$ as compared with the vehicle group using Student's t-test. Groups not treated with any drug or vehicle are indicated as 'No Tx'.

A

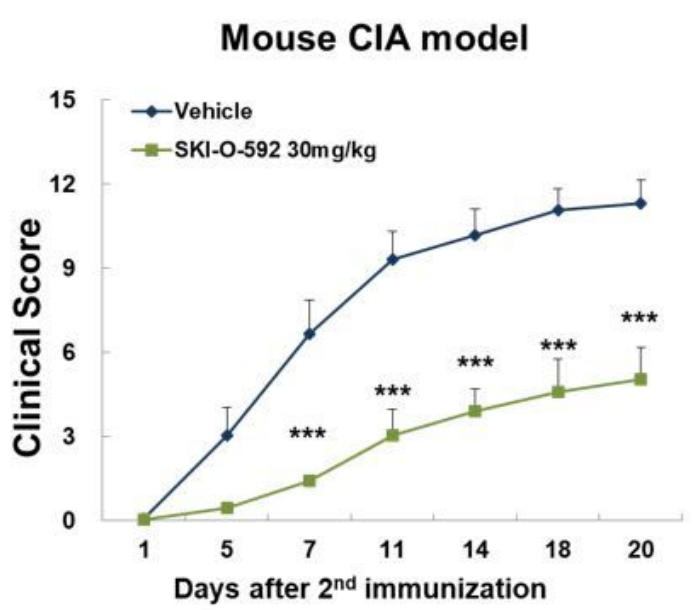

B

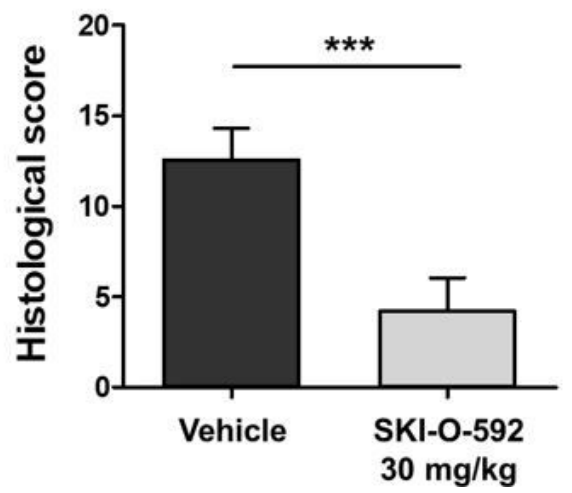

C

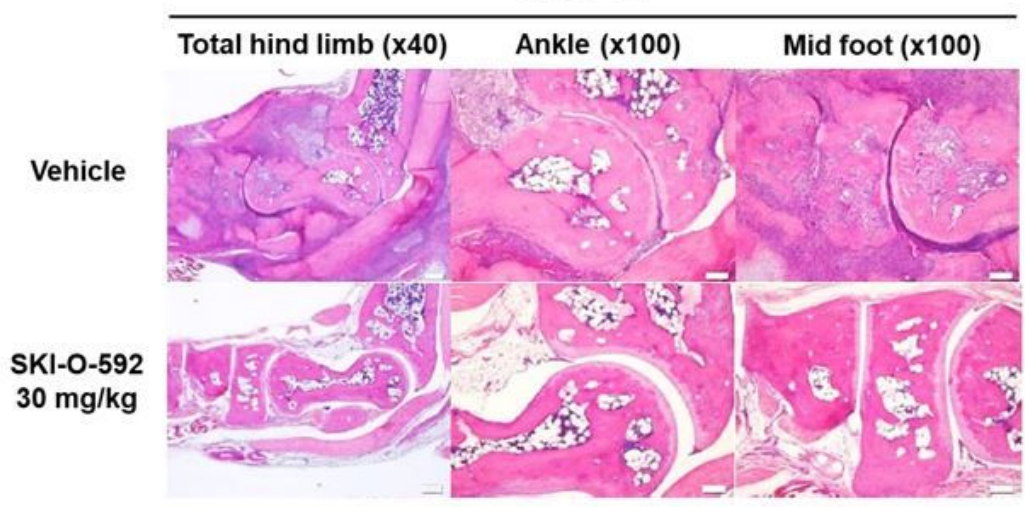

D

Safranin-O

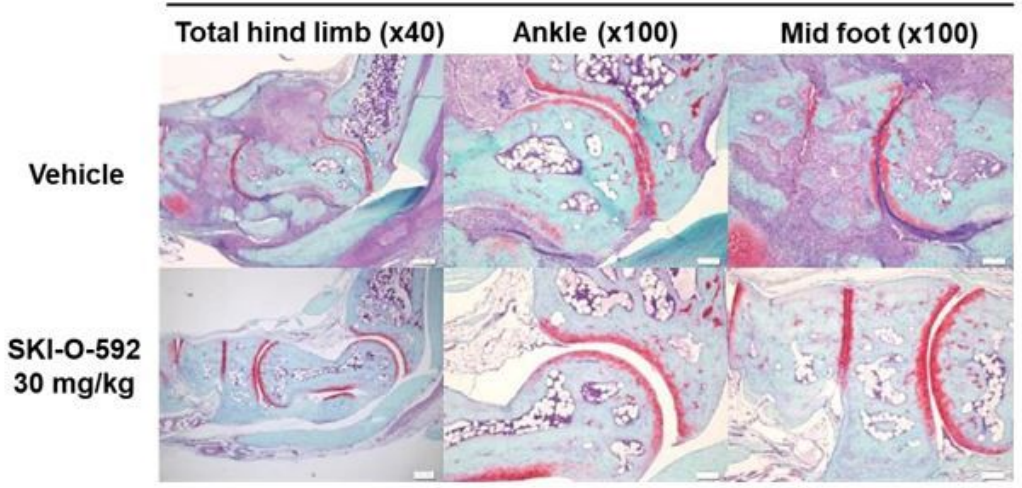

\section{Figure 8}

SKI-0-592 decreases clinical score and joint inflammation in a mouse CIA model. CIA was induced in DBA/1J mice. Vehicle $(n=10)$ or SKI-0-592 $(30 \mathrm{mg} / \mathrm{kg}, \mathrm{n}=10)$ was orally administered daily a week after CIA induction. (A) The clinical score of arthritis after day 7 was significantly decreased in SKI-0-592treated groups compared to the vehicle-treated group. (B) The histological score was also decreased in the SKI-O-592 treated group. Joint sections from animals treated with vehicle and drugs were stained with 
(C) H\&E, or (D) Safranin O. Data are presented as mean \pm SD. *** $p<0.001$, as compared with vehicle group using two-way ANOVA with Bonferroni correction.

A

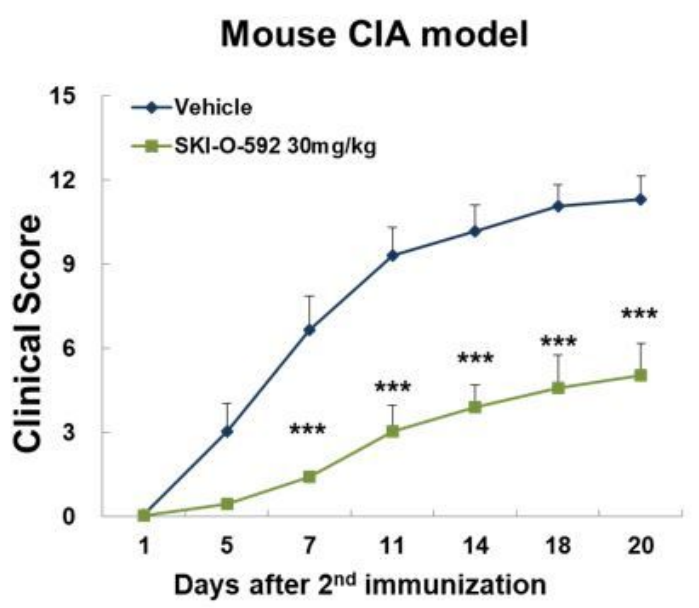

B

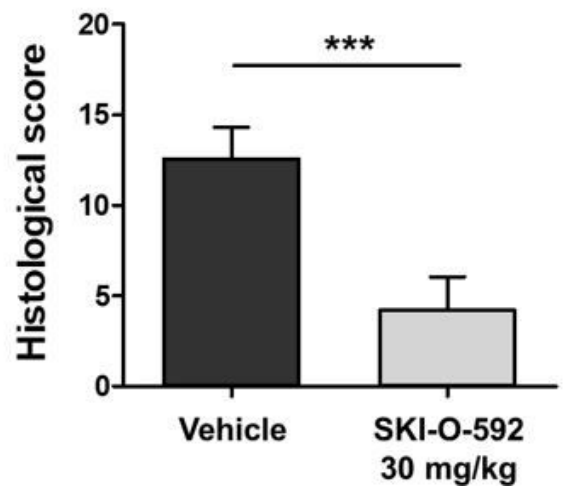

C

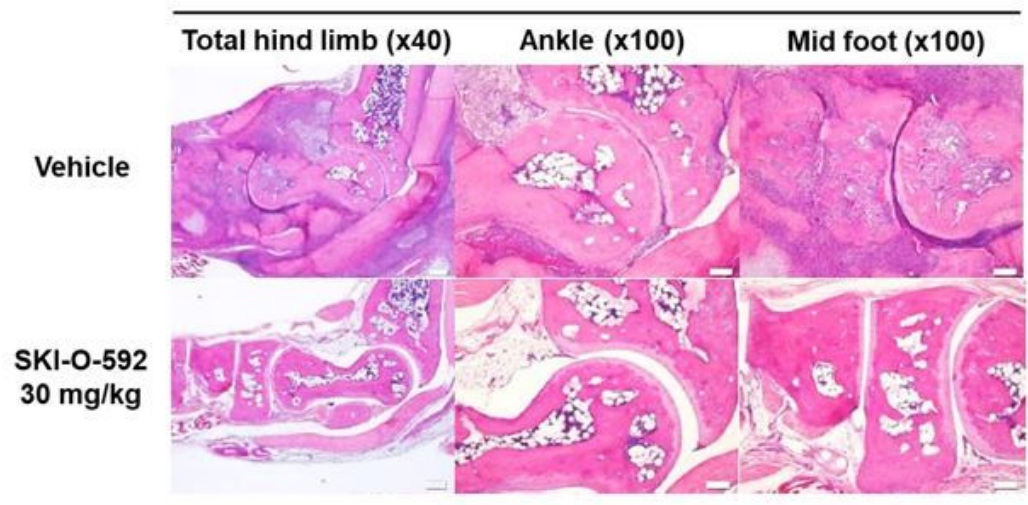

D

\section{Safranin-O}

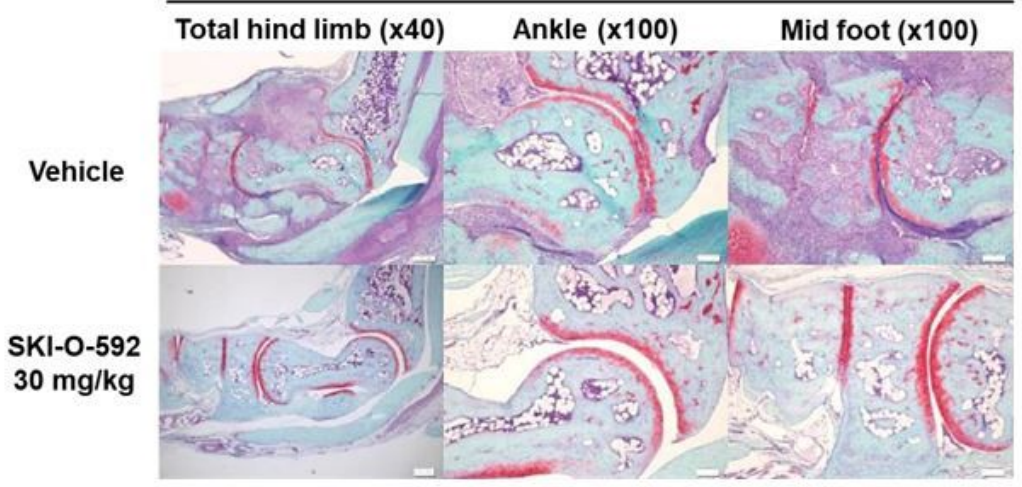

\section{Figure 8}

SKI-0-592 decreases clinical score and joint inflammation in a mouse CIA model. CIA was induced in DBA/1J mice. Vehicle $(n=10)$ or SKI-0-592 $(30 \mathrm{mg} / \mathrm{kg}, \mathrm{n}=10)$ was orally administered daily a week after $\mathrm{CIA}$ induction. (A) The clinical score of arthritis after day 7 was significantly decreased in SKI-0-592treated groups compared to the vehicle-treated group. (B) The histological score was also decreased in the SKI-0-592 treated group. Joint sections from animals treated with vehicle and drugs were stained with (C) H\&E, or (D) Safranin O. Data are presented as mean \pm SD. $* \star * ~ p<0.001$, as compared with vehicle group using two-way ANOVA with Bonferroni correction. 